\title{
EQUIVALENCE OF CATEGORIES BETWEEN COEFFICIENT SYSTEMS AND SYSTEMS OF IDEMPOTENTS
}

\author{
THOMAS LANARD
}

\begin{abstract}
The consistent systems of idempotents of Meyer and Solleveld allow to construct Serre subcategories of $\operatorname{Rep}_{R}(G)$, the category of smooth representations of a $p$-adic group $G$ with coefficients in $R$. In particular, they were used to construct level 0 decompositions when $R=\overline{\mathbb{Z}}_{\ell}, \ell \neq p$, by Dat for $\mathrm{GL}_{n}$ and the author for a more general group. Wang proved in the case of $\mathrm{GL}_{n}$ that the subcategory associated with a system of idempotents is equivalent to a category of coefficient systems on the Bruhat-Tits building. This result was used by Dat to prove an equivalence between an arbitrary level zero block of $\mathrm{GL}_{n}$ and a unipotent block of another group. In this paper, we generalize Wang's equivalence of category to a connected reductive group on a non-archimedean local field.
\end{abstract}

\section{INTRODUCTION}

Let $F$ be a non-archimedean local field and $G$ the $F$-points of a connected reductive group over $F$. Let $p$ be the characteristic of the residue field of $F$. Denote by BT the semi-simple Bruhat-Tits building of $G$. The building is a polysimplicial complex, partially ordered by the order relation $\sigma \leq \tau$ if $\sigma$ is a face of $\tau$.

Let $R$ be a commutative ring in which $p$ is invertible. A coefficient system $\Gamma$ on $\mathrm{BT}$ with coefficients in $R$ is a contravariant functor from the category $(\mathrm{BT}, \leq)$ to the category of $R$-modules. In other words, this is the data of $R$-modules $\left(V_{\sigma}\right)_{\sigma \in \mathrm{BT}}$ and $R$-morphisms $\varphi_{\tau}^{\sigma}: V_{\sigma} \rightarrow V_{\tau}$ if $\tau \leq \sigma$, such that $\varphi_{\sigma}^{\sigma}=\mathrm{Id}$, and $\varphi_{\omega}^{\tau} \circ \varphi_{\tau}^{\sigma}=\varphi_{\omega}^{\sigma}$ if $\omega \leq \tau \leq \sigma$. A coefficient system is said to be $G$-equivariant if for every $g \in G$ and for every $\sigma \in \mathrm{BT}$ there is an isomorphism $\alpha_{g, \sigma}: V_{\sigma} \rightarrow V_{g \sigma}$ compatible with the $\varphi_{\tau}^{\sigma}$ and such that $\alpha_{1, \sigma}=\mathrm{Id}, \alpha_{g, h \sigma} \circ \alpha_{h, \sigma}=\alpha_{g h, \sigma}$. To $\Gamma$ we associate a graduated cellular chain complex $C_{*}(\mathrm{BT}, \Gamma)=\bigoplus_{\sigma \in \mathrm{BT}} V_{\sigma}$ (see Section 1 for more details and the definition of the differential). The homology of $C_{*}(\mathrm{BT}, \Gamma)$ is then noted $H_{*}(\mathrm{BT}, \Gamma)$.

When $R=\mathbb{C}$, a fundamental example of coefficient systems is given by the work of Schneider and Stuhler [SS97. Let $\sigma \in \mathrm{BT}$ and let $G_{\sigma}^{\circ}$ be the parahoric subgroup of $G$ at $\sigma$. Schneider and Stuhler then constructed a filtration of $G_{\sigma}^{\circ}$ by compact open subgroups $G_{\sigma}^{\circ} \supseteq U_{\sigma}^{(0)} \supseteq U_{\sigma}^{(1)} \supseteq \cdots \supseteq U_{\sigma}^{(r)} \supseteq \cdots$. Then, they associate to $r \in \mathbb{N}$ and $V$ a smooth $R G$-module, the $G$-equivariant coefficient system $\sigma \mapsto V^{U_{\sigma}^{(r)}}$. If $V$ is genertated by $V^{U_{x}^{(r)}}$ for some vertex $x$, then the chain complex of this coefficient system provides a projective resolution of $V$.

Complex representations of $p$-adic groups were first studied. Then, in order to extend the local Langlands programme to representations with coefficients in a field,

Received by the editors August 4, 2020, and, in revised form, January 14, 2021, and February $8,2021$.

2020 Mathematics Subject Classification. Primary 22E50; Secondary 20E42. 
or a ring, as general as possible, it appeared interesting to study $R$-representations. This was first initiated by Vignéras in Vig96. She extended the natural projective resolutions of Schneider and Stuhler to representations on vector spaces over fields of characteristic not equal to $p$ in [Vig97.

Meyer and Solleveld generalize these process in [MS10]. Denote by $\mathcal{H}_{R}(G)$ the Hecke algebra of $G$ with coefficients in $R$. Let $\mathrm{BT}_{0}$ be the set of vertices in BT, that is the set of dimension 0 polysimplices. Let $e=\left(e_{\sigma}\right)_{\sigma \in \mathrm{BT}}$ be a system of idempotents of $\mathcal{H}_{R}(G)$ satisfying properties of consistency (see [MS10, Def 2.1] or Definition 1.1). Then the functor $\sigma \rightarrow e_{\sigma}(V)$ provides a $G$-equivariant coefficient system. They then showed that if we denote by $\operatorname{Rep}_{R}(G)$ the abelian category of smooth $G$ representations with coefficients in $R$ and $\operatorname{Rep}_{R}^{e}(G)$ the full subcategory of objects $V$ in $\operatorname{Rep}_{R}(G)$ such that $V=\sum_{x \in \mathrm{BT}_{0}} e_{x} V$, then $\operatorname{Rep}_{R}^{e}(G)$ is a Serre subcategory (MS10, Thm 3.1]). We recall that a Serre subcategory is a full subcategory $\mathfrak{S}$ of an Abelian category $\mathfrak{A}$ such that for every exact sequence $0 \rightarrow A \rightarrow B \rightarrow C$ in $\mathfrak{A}$ we have that $B \in \mathfrak{S}$ if and only if $A \in \mathfrak{S}$ and $C \in \mathfrak{S}$.

This result allowed Dat in Dat18 to reconstruct the depth 0 blocks of $\mathrm{GL}_{n}(F)$ for $R=\overline{\mathbb{Z}}_{\ell}$. Subsequently, decompositions on $\overline{\mathbb{Z}}_{\ell}$ were obtained for $G$ which splits over an unramified extension of $F$ in Lan18a] and Lan18b.

Let $e$ be a consistent system of idempotents. We also assume that if $x$ is a vertex of $\sigma$ then $e_{\sigma} \in \mathcal{H}_{R}\left(G_{x}\right)$, where $G_{x}$ is the pointwise stabilizer of $x$ in $G$. A $e$-coefficient system is a $G$-equivariant coefficient system such that $\varphi_{x}^{\sigma}: V_{\sigma} \rightarrow V_{x}$

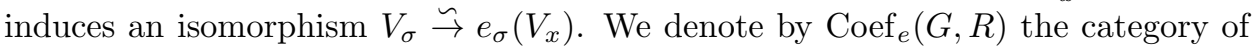
$e$-coefficient systems.

Theorem. Let $\Gamma$ be a e-coefficient system on $\mathrm{BT}$. Then, the chain complex $C_{*}(\mathrm{BT}$, $\Gamma)$ is exact except in degree 0.

For $V$ a smooth $R$-representation of $G$, let $\Gamma(V)$ be the $e$-coefficient system defined by $V_{\sigma}=e_{\sigma}(V)$. We then prove the following theorem

Theorem. The functor

$$
\begin{array}{ccc}
\operatorname{Rep}_{R}^{e}(G) & \rightarrow & \operatorname{Coef}_{e}(G, R) \\
V & \mapsto & \Gamma(V)
\end{array}
$$

admits a quasi-inverse $\Gamma \mapsto H_{0}(\mathrm{BT}, \Gamma)$, so induces an equivalence of categories.

Remark.

(1) In the case of $\mathrm{GL}_{n}(F)$, these results were proven by Wang in Wan17.

(2) The equivalence on $\mathrm{GL}_{n}(F)$ was used by Dat in Dat18 to construct an equivalence of categories between an arbitrary block of $\mathrm{GL}_{n}(F)$ and a unipotent block of another reductive $p$-adic group. Thus, we can hope that this theorem can be used to show an equivalence between blocks in a more general context.

(3) This theorem applies in particular to all the categories constructed in Lan18a] and Lan18b.

Schneider and Stuhler proved in [SS97, Thm. V.1] a general equivalence of categories between a category of representations and of coefficient systems. In SS97, Thm. V.1] the field of coefficient is $R=\mathbb{C}$. However, the main argument is the existence of coefficient systems $\Gamma(V)$, natural in $V$, that provide resolutions of $V$. For the coefficient systems considered in this article, the resolution properties are 
proven in [MS10, Thm. 2.4]. Hence the result of Schneider and Stuhler is valid in our context. The main difference is that, in the category of coefficient systems, they need to formally invert morphisms $s$ such that $H_{0}(\mathrm{BT}, s)$ is an isomorphism. One strategy to prove the theorem wanted here, could be to restrict the functor of Schneider and Stuhler to $\operatorname{Coef}_{e}(G, R)$ and prove that the localization is trivial. Proving that is similar to prove the results of this article (for instance, it follows from Theorem 4.10). Therefore, we will instead follow the strategy of Wan17. Moreover, this will also show different techniques to prove acyclicity of chain complexes, which is very important.

This paper is composed of four parts. The first one recalls the definitions of coefficient systems and systems of idempotents. The proof in Wan17 uses geometric properties of the Bruhat-Tits building specific to $\mathrm{GL}_{n}$, in particular, the notion of taut paths. These paths do not work well for a general reductive group, so we introduce in Section 2 a more flexible notion: the admissible paths. They allow us to redefine the local maps in Section 3 . The goal of this third section is to check that this definition is independent of the path chosen. Once this is done, we can easily show, in the last section, that the "new" local maps satisfy all the properties that we need to follow the rest of the proof of Wang.

\section{Coefficient systems And systems of idempotents}

Let $F$ be a non-archimedean local field and $G$ the $F$-points of a connected reductive group over $F$. Let $p$ be the characteristic of the residue field of $F$.

Denote by BT the semi-simple Bruhat-Tits building associated with $G$ (cf. BT72 and BT84]). The building is a polysimplicial complex and we denote by $\mathrm{BT}_{0}$ the set of dimension 0 polysimplices, that is the vertices. In the rest of this article we will use Latin letters $x, y, \cdots$ for the vertices and Greek letters $\sigma, \tau, \cdots$ for general polysimplices. The building BT is partially ordered by the partial order $\sigma \leq \tau$ if $\sigma$ is a face of $\tau$. A set of polysimplices $\sigma_{1}, \cdots, \sigma_{k}$ is said to be adjacent if there is a polysimplex $\sigma$ such as for all $i \in\{1, \cdots, k\}, \sigma_{i} \leq \sigma$. If $\left\{\sigma_{1}, \cdots, \sigma_{k}\right\}$ is a set of adjacent polysimplices we denote by $\left[\sigma_{1}, \cdots, \sigma_{k}\right]$ the smallest polysimplex containing $\sigma_{1} \cup \cdots \cup \sigma_{k}$. For $\sigma, \tau$ two polysimplices, denote by $H(\sigma, \tau)$ the polysimplicial hull of $\sigma$ and $\tau$, that is, the intersection of all the apartments containing $\sigma \cup \tau$.

Let $R$ be a commutative ring in which $p$ is invertible. A coefficient system $\Gamma$ on $\mathrm{BT}$ with coefficients in $R$ is a contravariant functor from the category $(\mathrm{BT}, \leq)$ to the category of $R$-modules. In other words, this is the data of $R$-modules $\left(V_{\sigma}\right)_{\sigma \in \mathrm{BT}}$ and $R$-morphisms $\varphi_{\tau}^{\sigma}: V_{\sigma} \rightarrow V_{\tau}$ for all polysimplices $\tau \leq \sigma$ such that: $\varphi_{\sigma}^{\sigma}=$ Id for all $\sigma \in \mathrm{BT}$, and $\varphi_{\omega}^{\tau} \circ \varphi_{\tau}^{\sigma}=\varphi_{\omega}^{\sigma}$ if $\omega, \tau, \sigma$ are polysimplicies such that $\omega \leq \tau \leq \sigma$.

To associate a cellular chain complex to $\Gamma$, each polysimplex is equipped with an orientation that induces an orientation on each of its faces. For all $\sigma, \tau \in \mathrm{BT}$, we define

$$
\epsilon_{\tau \sigma}= \begin{cases}1 & \text { if } \tau \leq \sigma \text { with compatible orientations } \\ -1 & \text { if } \tau \leq \sigma \text { with opposite orientations } \\ 0 & \text { if } \tau \text { is not a face of } \sigma\end{cases}
$$

Let $\Sigma$ be a subcomplex of BT. The cellular chain complex $C_{*}(\Sigma, \Gamma)$ on $\Sigma$ with coefficients $\Gamma$ is the $\mathbb{N}$-graduated chain complex

$$
C_{*}(\Sigma, \Gamma):=\cdots \stackrel{\partial}{\rightarrow} C_{c}^{o r}\left(\Sigma_{d}, \Gamma\right) \stackrel{\partial}{\rightarrow} \cdots \stackrel{\partial}{\rightarrow} C_{c}^{o r}\left(\Sigma_{0}, \Gamma\right)
$$


where $C_{c}^{o r}\left(\Sigma_{d}, \Gamma\right):=\bigoplus_{\sigma \in \Sigma, \operatorname{dim}(\sigma)=d} V_{\sigma}$ and the differential is given by

$$
\partial\left(\left(v_{\sigma}\right)_{\sigma \in \mathrm{BT}}\right)_{\tau}=\sum_{\sigma \in \Sigma} \epsilon_{\tau \sigma} \varphi_{\sigma}^{\tau}\left(v_{\sigma}\right)
$$

The homology of $C_{*}(\Sigma, \Gamma)$ is then denoted $H_{*}(\Sigma, \Gamma)$.

A coefficient system is said to be $G$-equivariant if for all $g \in G$ and $\sigma \in$ BT there is an isomorphism $\alpha_{g, \sigma}: V_{\sigma} \rightarrow V_{g \sigma}$ compatible with the $\varphi_{\tau}^{\sigma}$ and such that $\alpha_{1, \sigma}=\mathrm{Id}, \alpha_{g, h \sigma} \circ \alpha_{h, \sigma}=\alpha_{g h, \sigma}$.

We can construct $G$-equivariant coefficient systems from a smooth $R G$-module $V$ and a consistent system of idempotents. We fix a Haar measure on $G$ and denote by $\mathcal{H}_{R}(G)$ the Hecke algebra of $G$ with coefficients in $R$, that is the algebra of functions from $G$ to $R$ locally constant with compact support.

1.1. Definition. A system of idempotents $e=\left(e_{x}\right)_{x \in \mathrm{BT}_{0}}$ of $\mathcal{H}_{R}(G)$ is consistent if the following properties are satisfied :

(1) $e_{x} e_{y}=e_{y} e_{x}$ when $x$ and $y$ are adjacent.

(2) $e_{x} e_{z} e_{y}=e_{x} e_{y}$ when $z \in H(x, y)$ and $z$ is adjacent to $x$.

(3) $e_{g x}=g e_{x} g^{-1}$ for all $x \in \mathrm{BT}_{0}$ and $g \in G$.

If $e$ is a consistent system of idempotents and $V$ is a smooth $R G$-module, then the functor $\sigma \rightarrow e_{\sigma}(V)$ provides a $G$-equivariant coefficient system.

Let $e$ be a consistent system of idempotents on BT. We will say that $e$ satisfies the condition (买) if

(*)

For every vertex $x$ and every polysimplex $\sigma$ containing $x$ we have $e_{\sigma} \in \mathcal{H}_{R}\left(G_{x}\right)$

where $G_{x}$ designate the pointwise stabilizer $x$.

Then if $e$ satisfies (因) and if $\Gamma$ is a $G$-equivariant coefficient system, the condition (荬) ensures that $e_{\sigma}$ acts on $V_{x}$. A $e$-coefficient system is then a $G$-equivariant coefficient system such that for every $x \leq \sigma$ the morphism $\varphi_{x}^{\sigma}: V_{\sigma} \rightarrow V_{x}$ induces an isomorphism $V_{\sigma} \stackrel{\sim}{\rightarrow} e_{\sigma}\left(V_{x}\right)$. We denote by $\operatorname{Coef}_{e}(G, R)$ the category of $e$-coefficient systems.

Let $\operatorname{Rep}_{R}(G)$ be the abelian category of smooth $G$-representations with coefficients in $R$ and $\operatorname{Rep}_{R}^{e}(G)$ the full subcategory of objects $V$ in $\operatorname{Rep}_{R}(G)$ such that $V=\sum_{x \in \mathrm{BT}_{0}} e_{x} V$. Note that $\operatorname{Rep}_{R}^{e}(G)$ is a Serre subcategory by [MS10, Thm 3.1].

For $e$ a consistent system of idempotents satisfying the condition (函), we have just constructed a functor

$$
\begin{array}{ccc}
\operatorname{Rep}_{R}^{e}(G) & \rightarrow & \operatorname{Coef}_{e}(G, R) \\
V & \mapsto & \Gamma(V)
\end{array}
$$

where $\Gamma(V)$ is the functor $\sigma \rightarrow e_{\sigma}(V)$. We wish to show that this functor induces an equivalence of categories.

\section{Admissible PATHS}

To define his local maps, Wang uses in Wan17] the notion of taut paths ("chemins tendus" in French) between two vertices of the building. However, this definition works well for $\mathrm{GL}_{n}$ but not for a general reductive group. We can define taut paths for a general building, but properties as in [Wan17, Lem 2.2.5] are no longer true (the simplicial hull of two vertices is not, in general, the intersection of two cones). 
To have more flexibility than with taut path we introduce in this section the notion of admissible paths. They will allow, in Section 3 , to define the local maps for $G$.

2.1. Definition. Let $\tau, \sigma \in \mathrm{BT}$. A sequence of polysimplices $\tau_{0}, \cdots, \tau_{n} \in \mathrm{BT}$ is called an admissible path from $\tau$ to $\sigma$ if

(1) $\tau_{0}=\tau$ and $\tau_{n}=\sigma$

(2) $\forall i \in\{0, \cdots, n-1\}, \tau_{i+1} \in H\left(\tau_{i}, \sigma\right)$

(3) $\forall i \in\{0, \cdots, n-1\}, \tau_{i} \leq \tau_{i+1}$ or $\tau_{i+1} \leq \tau_{i}$

2.2. Remark. In Wan17, (2.2.4)], we have the definition of taut paths ("chemins tendus" in French). We can notice that if $\left(z_{0}, \cdots, z_{m}\right)$ is a taut path, then $\left(z_{0},\left[z_{0}, z_{1}\right]\right.$, $\left.z_{1},\left[z_{1}, z_{2}\right], \cdots, z_{m-1},\left[z_{m-1}, z_{m}\right], z_{m}\right)$ is an admissible path.

2.3. Lemma. Let $\tau_{0}, \cdots, \tau_{n} \in \mathrm{BT}$.

(1) If $\tau_{0}, \cdots, \tau_{n}$ is an admissible path then, for all $k \in\{0, \cdots, n\}, \tau_{k}, \cdots, \tau_{n}$ is also an admissible path.

(2) Let $k \in\{0, \cdots, n\}$ and assume that $\tau_{0}, \cdots, \tau_{k}$ and $\tau_{k}, \cdots, \tau_{n}$ are admissible paths. Then $\tau_{0}, \cdots, \tau_{n}$ is an admissible path if and only if for all $i \in$ $\{0, \cdots, k\}, \tau_{k} \in H\left(\tau_{i}, \tau_{n}\right)$.

(3) Let $k, l \in\{0, \cdots, n\}$ with $k \leq l$. Then if $\tau_{0}, \cdots, \tau_{n}$ is an admissible path and if $\tau_{k}, \cdots, \tau_{l}$ are adjacent then $\tau_{0}, \cdots, \tau_{k},\left[\tau_{k}, \cdots, \tau_{l}\right], \tau_{l}, \cdots, \tau_{n}$ is again an admissible path.

Proof.

(1) It is obvious.

(2) Let us assume that $\tau_{0}, \cdots, \tau_{n}$ is an admissible path. Then for any $i$, since $\tau_{i+1} \in H\left(\tau_{i}, \tau_{n}\right)$, we have $H\left(\tau_{i+1}, \tau_{n}\right) \subseteq H\left(\tau_{i}, \tau_{n}\right)$. So for every $i \leq k$, $\tau_{k} \in H\left(\tau_{i}, \tau_{n}\right)$.

Conversely, let us assume that for every $i \in\{0, \cdots, k\}, \tau_{k} \in H\left(\tau_{i}, \tau_{n}\right)$ and let us show that $\tau_{0}, \cdots, \tau_{n}$ is an admissible path. The only condition we have to check is that $\tau_{i+1} \in H\left(\tau_{i}, \sigma\right)$. This is immediate for $i \in\{k, \cdots, n-$ $1\}$ because $\tau_{k}, \cdots, \tau_{n}$ is an admissible path. For $i<k, \tau_{0}, \cdots, \tau_{k}$ being an admissible path, $\tau_{i+1} \in H\left(\tau_{i}, \tau_{k}\right)$. But $\tau_{k} \in H\left(\tau_{i}, \tau_{n}\right)$ so $H\left(\tau_{i}, \tau_{k}\right) \subseteq$ $H\left(\tau_{i}, \tau_{n}\right)$ and we have the result.

(3) All we have to do is to check the conditions of being an admissible path between $\tau_{k}$ and $\left[\tau_{k}, \cdots, \tau_{l}\right]$ and between $\left[\tau_{k}, \cdots, \tau_{l}\right]$ and $\tau_{l}$. First we have $\tau_{k} \leq$ $\left[\tau_{k}, \cdots, \tau_{l}\right]$ and $\tau_{l} \leq\left[\tau_{k}, \cdots, \tau_{l}\right]$. Since $\tau_{l} \leq\left[\tau_{k}, \cdots, \tau_{l}\right], \tau_{l} \in H\left(\left[\tau_{k}, \cdots, \tau_{l}\right], \tau_{n}\right)$. All that remains to be checked is that $\left[\tau_{k}, \cdots, \tau_{l}\right] \in H\left(\tau_{k}, \tau_{n}\right)$, that is, for any $i \in\{k, \cdots, l\}, \tau_{i} \in H\left(\tau_{k}, \tau_{n}\right)$. Now, this condition is satisfied by (2), which completes the proof.

2.4. Lemma. Let $\sigma, \tau, \omega \in \mathrm{BT}$ such that $\omega \in H(\sigma, \tau)$. Then there exists an admissible path $\tau_{0}=\tau, \cdots, \tau_{k}=\omega$ from $\tau$ to $\omega$ such that for all $i, \omega \in H\left(\tau_{i}, \sigma\right)$.

In particular, there is an admissible path $\tau_{0}=\tau, \cdots, \tau_{k}=\omega, \cdots, \tau_{n}=\sigma$ from $\tau$ to $\sigma$ such that $\tau_{0}, \cdots, \tau_{k}$ is an admissible path from $\tau$ to $\omega$.

Proof. The first assertion is MS10, Lem. 2.15] and the second one follows from (2). in Lemma 2.3 .

\section{THE LOCAL MAPS}

The admissible paths allow us to redefine the local maps of Wan17. The main purpose of this section will be to show that the definition given below is independent 
of the choice of the admissible path. This is the most technical section. Hence, before we start, we will give a sketch of the proof. The first step is to simplify the admissible path chosen, without changing the local map. For instance, if $x$ and $y$ are two adjacent vertices we want to simplify a path like $x,[x, y], x,[x, y], y$ into $x,[x, y], y$. This is done in Lemmas 3.3 and 3.5 and we prove the properties satisfied by this simpler path in Lemma 3.7. We continue our simplification process (mostly for technical reasons in the proof) and we show that we can transform an admissible path from $\tau$ to $\sigma$ so that the second vertex is the unique maximal polysimplex in $H(\tau, \sigma)$ adjacent to $\tau$ (Lemma 3.11). In this way, we can assume that $\tau$ is maximal. Now, we prove the result by induction. Since we start with a maximal polysimplex, the "size" of the path from the second vertex will be smaller and we can apply the induction hypothesis. Hence we can modify the path from the second vertex, and by choosing a suitable vertex in the middle we can again change the beginning of the path (this is done in Lemma 3.12). Hence we can "transform" a path into a "relatively close" one. By repeatedly applying these transformations we show in Proposition 3.14 that we can obtain any other admissible path from $\tau$ to $\omega$.

Let $e$ be a consistent system of idempotents which satisfies (函). Let $\Gamma=\left(V_{\sigma}\right)_{\sigma \in \mathrm{BT}}$ be a $e$-coefficient system. When $x \leq \sigma$, we identify $V_{\sigma}$ to $e_{\sigma}\left(V_{x}\right)$ via the morphism $\varphi_{x}^{\sigma}$. For two polysimplices $\tau, \sigma$ such that $\tau \leq \sigma$, we have $V_{\sigma}=e_{\sigma}\left(V_{\tau}\right)$ and we denote by $p_{\sigma}^{\tau}$ the projection $V_{\tau} \rightarrow e_{\sigma}\left(V_{\tau}\right)=V_{\sigma}$.

Let $\tau, \sigma \in \mathrm{BT}$ such that $\tau \leq \sigma$. Then we get two maps

$$
\begin{gathered}
p_{\sigma}^{\tau}: V_{\tau} \rightarrow e_{\sigma} V_{\tau}=V_{\sigma}, \\
\varphi_{\tau}^{\sigma}: V_{\sigma} \hookrightarrow V_{\tau} .
\end{gathered}
$$

Thus we define if $\tau \leq \sigma$ or $\sigma \leq \tau$

$$
\varepsilon_{\sigma}^{\tau}: V_{\tau} \rightarrow V_{\sigma}
$$

by $\varepsilon_{\sigma}^{\tau}=p_{\sigma}^{\tau}$ if $\tau \leq \sigma$ and $\varepsilon_{\sigma}^{\tau}=\varphi_{\sigma}^{\tau}$ if $\tau \geq \sigma$.

3.1. Definition. Let $\tau, \sigma \in \mathrm{BT}$. Take $\tau_{0}=\tau, \cdots, \tau_{n}=\sigma$ an admissible path between $\tau$ and $\sigma$. We define $\varepsilon_{\sigma}^{\tau}: V_{\tau} \rightarrow V_{\sigma}$ by

$$
\varepsilon_{\sigma}^{\tau}=\varepsilon_{\tau_{n}}^{\tau_{n-1}} \circ \cdots \circ \varepsilon_{\tau_{1}}^{\tau_{0}} .
$$

3.2. Remark. If $\left(z_{0}, \cdots, z_{m}\right)$ is a taut path according to Wan17, then the definition of $\varepsilon_{z_{m}}^{z_{0}}$ given in [Wan17, Section 2.2] is the same as the one given here, if we consider the admissible path $\left(z_{0},\left[z_{0}, z_{1}\right], z_{1}, \cdots, z_{m-1},\left[z_{m-1}, z_{m}\right], z_{m}\right)$.

The purpose of what follows is to show that this definition is independent of the admissible path chosen. We need preliminary lemmas.

3.3. Lemma. Let $\tau_{0}, \cdots, \tau_{n} \in \mathrm{BT}$ be polysimplices such that for all $i$ we have $\tau_{i} \leq \tau_{i+1}$ or $\tau_{i+1} \leq \tau_{i}$. We also assume that $\tau_{0}, \cdots, \tau_{n}$ are adjacent. Then

$$
\varepsilon_{\tau_{n}}^{\tau_{n-1}} \circ \cdots \circ \varepsilon_{\tau_{1}}^{\tau_{0}}=\varepsilon_{\tau_{n}}^{\left[\tau_{0}, \cdots, \tau_{n}\right]} \circ \varepsilon_{\left[\tau_{0}, \cdots, \tau_{n}\right]}^{\tau_{0}} .
$$

Proof. Let us show the result by induction on $n$.

The result is clear for $n=1$. So let us take $\tau_{0}, \cdots, \tau_{n}$ as in the statement and assume the result true for $n-1$. The path $\tau_{1}, \cdots, \tau_{n}$ verifies the conditions of the statement thus by the induction hypothesis

$$
\varepsilon_{\tau_{n}}^{\tau_{n-1}} \circ \cdots \circ \varepsilon_{\tau_{2}}^{\tau_{1}}=\varepsilon_{\tau_{n}}^{\left[\tau_{1}, \cdots, \tau_{n}\right]} \circ \varepsilon_{\left[\tau_{1}, \cdots, \tau_{n}\right]}^{\tau_{1}}
$$


and so

$$
\varepsilon_{\tau_{n}}^{\tau_{n-1}} \circ \cdots \circ \varepsilon_{\tau_{1}}^{\tau_{0}}=\varepsilon_{\tau_{n}}^{\left[\tau_{1}, \cdots, \tau_{n}\right]} \circ \varepsilon_{\left[\tau_{1}, \cdots, \tau_{n}\right]}^{\tau_{1}} \circ \varepsilon_{\tau_{1}}^{\tau_{0}} .
$$

There are two cases:

(1) If $\tau_{0} \leq \tau_{1}$, then $\varepsilon_{\tau_{n}}^{\tau_{n-1}} \circ \cdots \circ \varepsilon_{\tau_{1}}^{\tau_{0}}$ corresponds to the map

$$
V_{\tau_{0}} \rightarrow e_{\tau_{1}}\left(V_{\tau_{0}}\right)=V_{\tau_{1}} \rightarrow e_{\left[\tau_{1}, \cdots, \tau_{n}\right]}\left(V_{\tau_{1}}\right)=e_{\left[\tau_{1}, \cdots, \tau_{n}\right]}\left(e_{\tau_{1}}\left(V_{\tau_{0}}\right)\right)=V_{\left[\tau_{1}, \cdots, \tau_{n}\right]} \hookrightarrow V_{\tau_{n}} .
$$

But $e_{\left[\tau_{1}, \cdots, \tau_{n}\right]} e_{\tau_{1}}=e_{\left[\tau_{1}, \cdots, \tau_{n}\right]}$ and since $\tau_{0} \leq \tau_{1},\left[\tau_{1}, \cdots, \tau_{n}\right]=\left[\tau_{0}, \cdots, \tau_{n}\right]$ and we have the result.

(2) If $\tau_{0} \geq \tau_{1}$, then $\varepsilon_{\tau_{n}}^{\tau_{n-1}} \circ \cdots \circ \varepsilon_{\tau_{1}}^{\tau_{0}}$ corresponds to the map

$$
V_{\tau_{0}} \hookrightarrow e_{\tau_{0}}\left(V_{\tau_{1}}\right) \rightarrow e_{\left[\tau_{1}, \cdots, \tau_{n}\right]}\left(e_{\tau_{0}}\left(V_{\tau_{1}}\right)\right) \hookrightarrow V_{\tau_{n}} .
$$

But

$$
e_{\left[\tau_{1}, \cdots, \tau_{n}\right]}\left(e_{\tau_{0}}\left(V_{\tau_{1}}\right)\right)=e_{\left[\tau_{1}, \cdots, \tau_{n}\right]} e_{\tau_{0}}\left(V_{\tau_{0}}\right)
$$

because $V_{\tau_{0}}=e_{\tau_{0}}\left(V_{\tau_{1}}\right)$ and $e_{\left[\tau_{1}, \cdots, \tau_{n}\right]} e_{\tau_{0}}=e_{\left[\left[\tau_{1}, \cdots, \tau_{n}\right], \tau_{0}\right]}=e_{\left[\tau_{0}, \cdots, \tau_{n}\right]}$. So the first two arrows correspond to the map

$$
V_{\tau_{0}} \rightarrow e_{\left[\tau_{0}, \cdots, \tau_{n}\right]}\left(V_{\tau_{0}}\right)
$$

and we have the result.

3.4. Corollary. Let $\tau$ and $\sigma$ be adjacent polysimplices. Then $\varepsilon_{\sigma}^{\tau}$ does not depend on the admissible path chosen.

Proof. Let $\tau_{0}, \cdots, \tau_{n}$ be an admissible path between $\tau$ and $\sigma$. The polysimplices $\tau$ and $\sigma$ being adjacent, $H(\sigma, \tau)$ is the closure of $[\sigma, \tau]$. The path $\tau_{0}, \cdots, \tau_{n}$ being admissible, for all $i \in\{0, \cdots, n\}$, we have $\tau_{i} \in H(\sigma, \tau)$ and so $\tau_{i} \leq[\sigma, \tau]$. Therefore the polysimplices $\tau_{0}, \cdots, \tau_{n}$ are adjacent and $\left[\tau_{0}, \cdots, \tau_{n}\right] \leq[\sigma, \tau]$. Since $\sigma, \tau \leq$ $\left[\tau_{0}, \cdots, \tau_{n}\right]$, we have $[\sigma, \tau] \leq\left[\tau_{0}, \cdots, \tau_{n}\right]$ and so $\left[\tau_{0}, \cdots, \tau_{n}\right]=[\sigma, \tau]$. Lemma 3.3 tells us that $\varepsilon_{\sigma}^{\tau}=\varepsilon_{\sigma}^{[\sigma, \tau]} \circ \varepsilon_{[\sigma, \tau]}^{\tau}$ which is independent of the chosen path.

3.5. Lemma. Let $\tau_{0}, \cdots, \tau_{n} \in \mathrm{BT}$ and $k, l \in\{0, \cdots, n\}$ with $k \leq l$. Let us assume that $\tau_{0}, \cdots, \tau_{n}$ is an admissible path and that $\tau_{k}, \cdots, \tau_{l}$ are adjacent. Then

$$
\varepsilon_{\tau_{n}}^{\tau_{n-1}} \circ \cdots \circ \varepsilon_{\tau_{1}}^{\tau_{0}}=\varepsilon_{\tau_{n}}^{\tau_{n-1}} \circ \cdots \varepsilon_{\tau_{l+1}}^{\tau_{l}} \circ \varepsilon_{\tau_{l}}^{\left[\tau_{k}, \cdots, \tau_{l}\right]} \circ \varepsilon_{\left[\tau_{k}, \cdots, \tau_{l}\right]}^{\tau_{k}} \circ \varepsilon_{\tau_{k}}^{\tau_{k-1}} \circ \cdots \circ \varepsilon_{\tau_{1}}^{\tau_{0}} .
$$

Proof. Lemma 2.3 (3) ensures that $\tau_{0}, \cdots, \tau_{k},\left[\tau_{k}, \cdots, \tau_{l}\right], \tau_{l}, \cdots, \tau_{n}$ is an admissible path. Now

$$
\varepsilon_{\tau_{n}}^{\tau_{n-1}} \circ \cdots \circ \varepsilon_{\tau_{1}}^{\tau_{0}}=\varepsilon_{\tau_{n}}^{\tau_{n-1}} \circ \cdots \varepsilon_{\tau_{l+1}}^{\tau_{l}} \circ \varepsilon_{\tau_{l}}^{\tau_{l-1}} \circ \cdots \circ \varepsilon_{\tau_{k+1}}^{\tau_{k}} \circ \varepsilon_{\tau_{k}}^{\tau_{k-1}} \circ \cdots \circ \varepsilon_{\tau_{1}}^{\tau_{0}} .
$$

Since $\tau_{k}, \cdots, \tau_{l}$ are adjacent, Lemma 3.3 gives us

$$
\varepsilon_{\tau_{l}}^{\tau_{l-1}} \circ \cdots \circ \varepsilon_{\tau_{k+1}}^{\tau_{k}}=\varepsilon_{\tau_{l}}^{\left[\tau_{k}, \cdots, \tau_{l}\right]} \circ \varepsilon_{\left[\tau_{k}, \cdots, \tau_{l}\right]}^{\tau_{k}} .
$$

And we get the wanted result.

3.6. Definition. Let $\tau, \sigma \in$ BT. We define $\rho(\tau, \sigma)$ to be the number of polysimplices in $H(\tau, \sigma)$. 
3.7. Lemma. Let $\tau_{0}, \cdots, \tau_{n}$ be an admissible path and $\omega \in \mathrm{BT}$ such that for all $i$, $\tau_{n} \in H\left(\tau_{i}, \omega\right)$. Then there exists another admissible path $\tau_{0}^{\prime}, \cdots, \tau_{m}^{\prime}$ such that

(1) $\tau_{0}^{\prime}=\tau_{0}$ and $\tau_{m}^{\prime}=\tau_{n}$

(2) $\varepsilon_{\tau_{n}}^{\tau_{n-1}} \circ \cdots \circ \varepsilon_{\tau_{1}}^{\tau_{0}}=\varepsilon_{\tau_{m}^{\prime}}^{\tau_{m-1}^{\prime}} \circ \cdots \circ \varepsilon_{\tau_{1}^{\prime}}^{\tau_{0}^{\prime}}$

(3) if $\tau_{i}^{\prime}>\tau_{i+1}^{\prime}$ and $i+1<m$ then $\rho\left(\tau_{i+1}^{\prime}, \omega\right)<\rho\left(\tau_{i}^{\prime}, \omega\right)$

(4) For all $i, \tau_{m}^{\prime} \in H\left(\tau_{i}^{\prime}, \omega\right)$

Proof. Let $k_{1}=\max \left\{i>0 \mid \tau_{0}, \cdots, \tau_{i}\right.$ are adjacent $\}$ (this set is non empty because $\tau_{1}$ and $\tau_{0}$ are adjacent). Likewise, if $k_{i}$ is defined and different from $n$, we define $k_{i+1}=\max \left\{j>k_{i} \mid \tau_{k_{i}}, \cdots, \tau_{j}\right.$ are adjacent $\}$. So we have just defined $m^{\prime}$ integers, $k_{1}, \cdots, k_{m^{\prime}}$ with $k_{m^{\prime}}=n$. Let $k_{0}=0$ and $m=2 m^{\prime}$.

Let us define for $i \in\left\{0, \cdots, m^{\prime}\right\}, \tau_{2 i}^{\prime}=\tau_{k_{i}}$, and for $i \in\left\{0, \cdots, m^{\prime}-1\right\}, \tau_{2 i+1}^{\prime}=$ $\left[\tau_{k_{i}}, \cdots, \tau_{k_{i+1}}\right]$.

Lemma 2.3 (3) applied $m^{\prime}$ times ensures that $\tau_{0}^{\prime}, \cdots, \tau_{m}^{\prime}$ is an admissible path. Let us verify that this path satisfies the requested properties.

(1) It is clear that $\tau_{0}^{\prime}=\tau_{0}$ and $\tau_{m}^{\prime}=\tau_{n}$.

(2) Lemma 3.5 applied $m^{\prime}$ times gives us the result.

(3) We can only have $\tau_{i}^{\prime}>\tau_{i+1}^{\prime}$ if $i=2 l+1$ is odd. So all we have to do is to show that $H\left(\tau_{2 l+2}^{\prime}, \omega\right) \varsubsetneqq H\left(\tau_{2 l+1}^{\prime}, \omega\right)$.

By definition of $k_{l+1}, \tau_{k_{l+1}+1}$ and $\tau_{2 l+1}^{\prime}$ are not adjacent $\left(\tau_{k_{l+1}+1}\right.$ exists because by hypothesis $i+1<m)$. Take $\mathcal{A}$ an apartment containing $H\left(\tau_{k_{l}}, \omega\right)$. By hypothesis $\tau_{n} \in H\left(\tau_{k_{l}}, \omega\right)$ and so $H\left(\tau_{k_{l}}, \tau_{n}\right) \subseteq H\left(\tau_{k_{l}}, \omega\right)$. Hence $\tau_{k_{l}}, \cdots, \tau_{n} \in \mathcal{A}$. Since $\tau_{k_{l+1}+1}$ and $\tau_{2 l+1}^{\prime}$ are not adjacent, there exists an affine root $a$ which separates $\tau_{k_{l+1}+1}$ and $\tau_{2 l+1}^{\prime}$. Let us say for example that $a_{\mid \tau_{k_{l+1}+1}}>0$ and $a_{\mid \tau_{2 l+1}^{\prime}}<0$. Notice that $\tau_{k_{l+1}+1} \in$ $H\left(\tau_{k_{l+1}}, \tau_{n}\right) \subseteq H\left(\tau_{k_{l+1}}, \omega\right)=H\left(\tau_{2 l+2}^{\prime}, \omega\right) \subseteq H\left(\tau_{2 l+1}^{\prime}, \omega\right)$. Hence if $a_{\mid \omega}<0$, since $\tau_{k_{l+1}+1} \in H\left(\tau_{2 l+1}^{\prime}, \omega\right)$ we would get that $a_{\mid \tau_{k_{l+1}+1}} \leq 0$ which is not. So $a_{\mid \omega} \geq 0$ and $a$ is an affine root which separates $\tau_{2 l+1}^{\prime}$ from $H\left(\tau_{k_{l+1}+1}, \omega\right)$ and so $\tau_{2 l+1}^{\prime} \notin H\left(\tau_{k_{l+1}+1}, \omega\right)$. But $\tau_{k_{l+1}+1} \leq \tau_{k_{l+1}}$ or $\tau_{k_{l+1}+1} \geq \tau_{k_{l+1}}$. We cannot have $\tau_{k_{l+1}+1} \leq \tau_{k_{l+1}}$ because if not $\tau_{k_{l}}, \cdots, \tau_{k_{l+1}+1}$ would be adjacent. So $\tau_{k_{l+1}+1} \geq \tau_{k_{l+1}}$. Thus $H\left(\tau_{k_{l+1}+1}, \omega\right) \supseteq H\left(\tau_{k_{l+1}}, \omega\right)=H\left(\tau_{2 l+2}^{\prime}, \omega\right)$. So $\tau_{2 l+1}^{\prime} \notin H\left(\tau_{2 l+2}^{\prime}, \omega\right)$ which completes the proof.

(4) We wish to show that for every $i, \tau_{n} \in H\left(\tau_{i}^{\prime}, \omega\right)$. If $i=2 l$ is even, the result is immediate since $\tau_{2 l}^{\prime}=\tau_{k_{l}}$. We are left with the case $i=2 l+1$ odd, that is, we want to show that $\tau_{n} \in H\left(\left[\tau_{k_{l}}, \cdots, \tau_{k_{l+1}}\right], \omega\right)$. But $\tau_{k_{l}} \leq\left[\tau_{k_{l}}, \cdots, \tau_{k_{l+1}}\right]$ so $H\left(\tau_{k_{l}}, \omega\right) \subseteq H\left(\left[\tau_{k_{l}}, \cdots, \tau_{k_{l+1}}\right], \omega\right)$ and $\tau_{n} \in H\left(\left[\tau_{k_{l}}, \cdots, \tau_{k_{l+1}}\right], \omega\right)$.

3.8. Lemma. Let $\tau, \sigma \in \mathrm{BT}$. Then there exists a unique maximal polysimplex $\bar{\tau} \in H(\tau, \sigma)$ with $\tau \leq \bar{\tau}$. That is, a polysimplex $\omega$ satisfies $\omega \in H(\tau, \sigma)$ and $\tau \leq \omega$ if and only if $\tau \leq \omega \leq \bar{\tau}$.

Proof. The proof is identical to Lemma 2.9 in MS10 by replacing $x$ with $\sigma$.

3.9. Lemma. Let $\tau, \sigma, \bar{\tau}$ like in Lemma 3.8. Let $\mathcal{A}$ be an apartment containing $H(\sigma, \tau)$ and $a$ an affine root. Then if $a_{\mid \bar{\tau}}=0$ we get $a_{\mid H(\sigma, \tau)}=0$.

Proof. Assume that $a_{\mid \bar{\tau}}=0$. Denote by $H=\operatorname{ker}(a)$ an affine hyperplane of $\mathcal{A}$. We then need to show that $H(\sigma, \tau) \subseteq H$. Let us prove the result by contradiction and assume that $H(\sigma, \tau) \nsubseteq H$. Take $x \in H(\sigma, \tau) \backslash H$. 
Let $\varphi:[0,1] \rightarrow$ BT be a geodesic between an interior point of $\bar{\tau}$ and $x$. Since $\varphi(0) \in H$ if there were $t \in] 0,1]$ such that $\varphi(t) \in H$, we would have that for every $t \in[0,1], \varphi(t) \in H$ and in particular that $x \in H$ which is not. So $\varphi(] 0,1]) \cap H=$ $\emptyset$. Each $\varphi(t)$ belongs to a polysimplex $\tau(t)$ and the map $t \mapsto \tau(t)$ is piecewise constant, so we can chose $t_{0}$ the smallest positive real where we have a jump. Let $\omega=\tau\left(t_{0} / 2\right)$. We must have $\bar{\tau} \leq \omega$ or $\omega \leq \bar{\tau}$. But $\omega \nsubseteq H$ and $\bar{\tau} \subseteq H$ so $\bar{\tau}<\omega$. But $\varphi\left(t_{0} / 2\right) \in H(\tau, \sigma)$ so $\omega \in H(\tau, \sigma)$ which contradicts the definition of $\bar{\tau}$.

3.10. Lemma. Let $\tau, \omega, \sigma \in \mathrm{BT}$ be polysimplices with $\omega \in H(\tau, \sigma)$. Take, as in Lemma 3.8, $\bar{\tau}$ maximal in $H(\tau, \sigma)$ such that $\tau \leq \bar{\tau}$ and $\bar{\omega}$ maximal in $H(\omega, \sigma)$ such that $\omega \leq \bar{\omega}$. Then $\bar{\tau} \in H(\tau, \bar{\omega})$.

Proof. Denote by $\bar{\tau}^{\prime}$ the maximal polysimplex of $H(\tau, \bar{\omega})$ such that $\tau \leq \bar{\tau}^{\prime}$. Then $H(\tau, \bar{\omega}) \subseteq H(\tau, \sigma)$ and so $\bar{\tau}^{\prime} \leq \bar{\tau}$.

Let us assume that $\bar{\tau}^{\prime} \neq \bar{\tau}$. Then $\bar{\tau}^{\prime}$ is a face of $\bar{\tau}$ and if we take $\mathcal{A}$ an apartment containing $H(\tau, \sigma)$ there exists an affine root $a$ such that $a_{\mid \bar{\tau}^{\prime}}=0$ and $a_{\mid \bar{\tau}}>0$. Since $a_{\mid \bar{\tau}^{\prime}}=0$ by Lemma $3.9 a_{\mid H(\tau, \bar{\omega})}=0$ and in particular $a_{\mid \tau}=0$ and $a_{\mid \bar{\omega}}=0$. Again by Lemma 3.9. since $a_{\mid \bar{\omega}}=0$ we have $a_{\mid H(\omega, \sigma)}=0$ and so $a_{\mid \sigma}=0$. Thus $a_{\mid \tau}=0$ and $a_{\mid \sigma}=0$ so $a_{\mid H(\tau, \sigma)}=0$. But $\bar{\tau} \in H(\tau, \sigma)$, so $a_{\mid \bar{\tau}}=0$ which is absurd.

3.11. Lemma. Let $\tau, \sigma \in \mathrm{BT}$. Take $\bar{\tau}$ maximal in $H(\tau, \sigma)$ such that $\tau \leq \bar{\tau}$. Let $\tau_{0}=\tau, \cdots, \tau_{n}=\sigma$ an admissible path between $\tau$ and $\sigma$. Then $\tau, \bar{\tau}, \tau_{0}, \cdots, \tau_{n}$ is again an admissible path and

$$
\varepsilon_{\tau_{n}}^{\tau_{n-1}} \circ \cdots \circ \varepsilon_{\tau_{1}}^{\tau_{0}}=\varepsilon_{\tau_{n}}^{\tau_{n-1}} \circ \cdots \circ \varepsilon_{\tau_{1}}^{\tau_{0}} \circ \varepsilon_{\tau_{0}}^{\bar{\tau}} \circ \varepsilon_{\bar{\tau}}^{\tau} .
$$

Proof. We have that $\tau \leq \bar{\tau}$ and so $H(\tau, \sigma) \subseteq H(\bar{\tau}, \sigma)$. Then since $\bar{\tau} \in H(\tau, \sigma)$ we deduce that $H(\bar{\tau}, \sigma) \subseteq H(\tau, \sigma)$. Therefore $H(\tau, \sigma)=H(\bar{\tau}, \sigma)$ and $\tau, \bar{\tau}, \tau_{0}, \cdots, \tau_{n}$ is an admissible path.

Let us show by induction on $n$ that for every polysimplices $\sigma$ and $\tau$ and for every admissible path $\tau_{0}, \cdots, \tau_{n}$ between $\tau$ and $\sigma$ that $\varepsilon_{\tau_{n}}^{\tau_{n-1}} \circ \cdots \circ \varepsilon_{\tau_{1}}^{\tau_{0}}=\varepsilon_{\tau_{n}}^{\tau_{n-1}} \circ \cdots \circ \varepsilon_{\tau_{1}}^{\tau_{0}} \circ$ $\varepsilon_{\tau_{0}}^{\bar{\tau}} \circ \varepsilon_{\bar{\tau}}^{\tau}$. The case $n=1$ is obvious. So let us assume the result true for $n-1$ and let us prove it for $n$.

Let $\varphi=\varepsilon_{\tau_{n}}^{\tau_{n-1}} \circ \cdots \circ \varepsilon_{\tau_{1}}^{\tau_{0}}$. The map $\varepsilon_{\tau_{n}}^{\tau_{n-1}} \circ \cdots \circ \varepsilon_{\tau_{1}}^{\tau_{0}} \circ \varepsilon_{\tau_{0}}^{\bar{\tau}} \circ \varepsilon_{\bar{\tau}}^{\tau}$ corresponds to the map

$$
V_{\tau} \stackrel{p_{\bar{\tau}}^{\tau}}{\rightarrow} V_{\bar{\tau}} \stackrel{\varphi_{\tau}^{\bar{\tau}}}{\rightarrow} V_{\tau} \stackrel{\varphi}{\rightarrow} V_{\sigma} .
$$

Let $v \in V_{\tau}$, we wish to show that $\varphi(v)=\varphi\left(e_{\bar{\tau}}(v)\right)$. The result we wish to demonstrate is therefore equivalent to saying that if $u \in V_{\tau}$ is such that $e_{\bar{\tau}}(u)=0$ then $\varphi(u)=0$.

Let $\psi=\varepsilon_{\tau_{n}}^{\tau_{n-1}} \circ \cdots \circ \varepsilon_{\tau_{2}}^{\tau_{1}}$. Denote by $\overline{\tau_{1}}$ the maximal polysimplex of $H\left(\tau_{1}, \sigma\right)$ such that $\tau_{1} \leq \overline{\tau_{1}}$. Then by the induction hypothesis, we know that if $v \in V_{\tau_{1}}$ is such that $e_{\overline{\tau_{1}}}(v)=0, \psi(v)=0$. Let $u \in V_{\tau}$ such that $e_{\bar{\tau}}(u)=0$, since $\varphi=\psi \circ \varepsilon_{\tau_{1}}^{\tau_{0}}$, all we have to do is show that $e_{\overline{\tau_{1}}}\left(\varepsilon_{\tau_{1}}^{\tau_{0}}(u)\right)=0$. There are two cases:

- $\tau_{0} \leq \tau_{1}$

Then $\tau_{1} \in H\left(\tau_{0}, \sigma\right)$ and $\tau_{0} \in H\left(\tau_{1}, \sigma\right)$ so $H\left(\tau_{0}, \sigma\right)=H\left(\tau_{1}, \sigma\right)$. Therefore $\overline{\tau_{1}}=\bar{\tau}$. Thus $e_{\overline{\tau_{1}}}\left(\varepsilon_{\tau_{1}}^{\tau_{0}}(u)\right)=e_{\bar{\tau}}\left(\varepsilon_{\tau_{1}}^{\tau_{0}}(u)\right)=\varepsilon_{\tau_{1}}^{\tau_{0}}\left(e_{\bar{\tau}} u\right)=0$.

- $\tau_{0} \geq \tau_{1}$

Then $\varepsilon_{\tau_{1}}^{\tau_{0}}=\varphi_{\tau_{1}}^{\tau_{0}}: V_{\tau_{0}} \hookrightarrow V_{\tau_{1}}$ and $e_{\overline{\tau_{1}}}\left(\varepsilon_{\tau_{1}}^{\tau_{0}}(u)\right)=e_{\overline{\tau_{1}}} e_{\tau}\left(\varepsilon_{\tau_{1}}^{\tau_{0}}(u)\right)$. But $\bar{\tau} \in H\left(\tau, \overline{\tau_{1}}\right)$ by Lemme 3.10, so Proposition 2.2 (e) of [MS10] tells us that $e_{\overline{\tau_{1}}} e_{\tau}=e_{\overline{\tau_{1}}} e_{\bar{\tau}} e_{\tau}=e_{\overline{\tau_{1}}} e_{\tau} e_{\bar{\tau}}$ and therefore $e_{\overline{\tau_{1}}}\left(\varepsilon_{\tau_{1}}^{\tau_{0}}(u)\right)=e_{\overline{\tau_{1}}} e_{\tau} e_{\bar{\tau}}\left(\varepsilon_{\tau_{1}}^{\tau_{0}}(u)\right)=$ $e_{\overline{\tau_{1}}} e_{\tau}\left(\varepsilon_{\tau_{1}}^{\tau_{0}}\left(e_{\bar{\tau}} u\right)\right)=0$. 
Let $\mathcal{P}(n)$ be the property that for every $\tau, \sigma \in \mathrm{BT}$ such that $\rho(\tau, \sigma) \leq n$ then $\varepsilon_{\sigma}^{\tau}$ does not depend on the choice of the admissible path.

3.12. Lemma. Let $\tau, \sigma \in \mathrm{BT}$ such that $\rho(\tau, \sigma)>1$. Let $\tau_{0}, \cdots, \tau_{n}$ and $\tau_{0}^{\prime}, \cdots, \tau_{m}^{\prime}$ be two admissible paths between $\tau$ and $\sigma$. We assume that

- $\mathcal{P}(\rho(\tau, \sigma)-1)$ is true

- $\rho\left(\tau_{1}, \sigma\right)<\rho(\tau, \sigma)$ and $\rho\left(\tau_{1}^{\prime}, \sigma\right)<\rho(\tau, \sigma)$

- There exists $\omega \in H\left(\tau_{1}, \sigma\right) \cap H\left(\tau_{1}^{\prime}, \sigma\right)$

- $\rho(\tau, \omega)<\rho(\tau, \sigma)$

- $\tau_{1} \in H(\tau, \omega)$ and $\tau_{1}^{\prime} \in H(\tau, \omega)$

Then

$$
\varepsilon_{\tau_{n}}^{\tau_{n-1}} \circ \cdots \circ \varepsilon_{\tau_{1}}^{\tau_{0}}=\varepsilon_{\tau_{m}^{\prime}}^{\tau_{m-1}^{\prime}} \circ \cdots \circ \varepsilon_{\tau_{1}^{\prime}}^{\tau_{0}^{\prime}} .
$$

Proof. $\varepsilon_{\tau_{n}}^{\tau_{n-1}} \circ \cdots \circ \varepsilon_{\tau_{1}}^{\tau_{0}}=\left(\varepsilon_{\tau_{n}}^{\tau_{n-1}} \circ \cdots \circ \varepsilon_{\tau_{2}}^{\tau_{1}}\right) \circ \varepsilon_{\tau_{1}}^{\tau_{0}}$. Since $\rho\left(\tau_{1}, \sigma\right)<\rho(\tau, \sigma)$ and that $\mathcal{P}(\rho(\tau, \sigma)-1)$ is true, $\varepsilon_{\tau_{n}}^{\tau_{n-1}} \circ \cdots \circ \varepsilon_{\tau_{2}}^{\tau_{1}}=\varepsilon_{\sigma}^{\tau_{1}}$ does not depend on the admissible path. Moreover $\omega \in H\left(\tau_{1}, \sigma\right)$ hence by Lemma 2.4 there exists an admissible path between $\tau_{1}$ and $\sigma$ going through $\omega$ such that the paths from $\tau_{1}$ to $\omega$ and from $\omega$ to $\sigma$ are admissible. Hence $\varepsilon_{\sigma}^{\tau_{1}}=\varepsilon_{\sigma}^{\omega} \circ \varepsilon_{\omega}^{\tau_{1}}$. Thus we have $\varepsilon_{\tau_{n}}^{\tau_{n-1}} \circ \cdots \circ \varepsilon_{\tau_{1}}^{\tau_{0}}=\varepsilon_{\sigma}^{\omega} \circ \varepsilon_{\omega}^{\tau_{1}} \circ \varepsilon_{\tau_{1}}^{\tau_{0}}$. Since $\rho(\tau, \omega)<\rho(\tau, \sigma)$ and that $\mathcal{P}(\rho(\tau, \sigma)-1)$ is true, $\varepsilon_{\omega}^{\tau}$ does not depend on the admissible path. In particular, since $\tau_{1} \in H(\tau, \omega)$, we can complete the path $\tau_{0}, \tau_{1}$ in an admissible path between $\tau$ and $\omega$. The path from $\tau_{1}$ to $\omega$ is then also admissible and the same goes for the path $\tau_{0}, \tau_{1}$. Thus $\varepsilon_{\omega}^{\tau_{1}} \circ \varepsilon_{\tau_{1}}^{\tau_{0}}=\varepsilon_{\omega}^{\tau_{0}}$. So we have just shown that

$$
\varepsilon_{\tau_{n}}^{\tau_{n-1}} \circ \cdots \circ \varepsilon_{\tau_{1}}^{\tau_{0}}=\varepsilon_{\sigma}^{\omega} \circ \varepsilon_{\omega}^{\tau} .
$$

But the assumptions about the paths $\tau_{0}, \cdots, \tau_{n}$ and $\tau_{0}^{\prime}, \cdots, \tau_{m}^{\prime}$ are symmetric, so we also have $\varepsilon_{\tau_{m}^{\prime}}^{\tau_{m-1}^{\prime}} \circ \cdots \circ \varepsilon_{\tau_{1}^{\prime}}^{\tau_{0}^{\prime}}=\varepsilon_{\sigma}^{\omega} \circ \varepsilon_{\omega}^{\tau}$. And finally

$$
\varepsilon_{\tau_{n}}^{\tau_{n-1}} \circ \cdots \circ \varepsilon_{\tau_{1}}^{\tau_{0}}=\varepsilon_{\tau_{m}^{\prime}}^{\tau_{m-1}^{\prime}} \circ \cdots \circ \varepsilon_{\tau_{1}^{\prime}}^{\tau_{0}^{\prime}}
$$

3.13. Lemma. Let $\tau, \sigma \in \mathrm{BT}$. Take $\mathcal{A}$ an apartment containing $H(\sigma, \tau)$. We assume that there exists an affine root a such that $a_{\left.\right|_{\tau}}>0$ and $a_{\left.\right|_{\sigma}}<0$. Then if $\omega \in H(\tau, \sigma)$ is a polysimplex containing an interior point $x$ with $a(x)=0$, we get $\rho(\omega, \sigma)<\rho(\tau, \sigma)$ and $\rho(\tau, \omega)<\rho(\tau, \sigma)$.

Proof. Since the situation is symmetrical in $\tau$ and $\sigma$, all we have to do is show that $\rho(\omega, \sigma)<\rho(\tau, \sigma)$. Since $\omega \in H(\tau, \sigma)$ we have $H(\omega, \sigma) \subseteq H(\tau, \sigma)$ and $\rho(\omega, \sigma) \leq$ $\rho(\tau, \sigma)$. Hence we need to prove that $H(\omega, \sigma) \varsubsetneqq H(\tau, \sigma)$.

Since $a(x)=0$ and $x$ is an interior point of $\omega$ we have $a_{\mid \omega}=0$. Hence $a_{\mid \omega} \leq 0$, $a_{\mid \sigma}<0$ and $a_{\mid \tau}>0$ so $a$ separates $\tau$ from $\omega$ and $\sigma$ and therefore $\tau \notin H(\omega, \sigma)$ which ends the proof.

3.14. Proposition. Let $\tau, \sigma \in \mathrm{BT}$. Then $\varepsilon_{\sigma}^{\tau}$ does not depend on the choice of the admissible path.

Proof. We prove the result by induction on $\rho(\tau, \sigma)$. The result is true if $\rho(\tau, \sigma)=1$. Then we assume that $\rho(\tau, \sigma)>1$ and that $\mathcal{P}(\rho(\tau, \sigma)-1)$ is true.

Let us then take two admissible paths $\tau_{0}, \cdots, \tau_{n}$ and $\tau_{0}^{\prime}, \cdots, \tau_{m}^{\prime}$. Lemma 3.11 allows us to assume that $\tau=\bar{\tau}$ where $\bar{\tau}$ is the maximal polysimplex of $H(\tau, \sigma)$ such that $\tau \leq \bar{\tau}$. By applying Lemma 3.7 (with $\omega=\sigma$ ) we can assume that $\tau_{0}, \cdots, \tau_{n}$ 
and $\tau_{0}^{\prime}, \cdots, \tau_{m}^{\prime}$ verify the conditions of the latter. Moreover, by removing the first simplices if they are equal, we can assume that $\tau_{0} \neq \tau_{1}$ and $\tau_{0}^{\prime} \neq \tau_{1}^{\prime}$. In particular we have that $\rho\left(\tau_{1}, \sigma\right)<\rho(\tau, \sigma)$ and $\rho\left(\tau_{1}^{\prime}, \sigma\right)<\rho(\tau, \sigma)$. The case where $\tau$ and $\sigma$ are adjacent is settled by Corollary 3.4 so we assume that $\tau$ and $\sigma$ are not adjacent.

Let $\mathcal{A}$ be an apartment containing $H(\tau, \sigma)$. Since $\tau$ and $\sigma$ are two polysimplices non adjacent, there exists an affine root $a$ such that $a_{\mid \tau}>0$ and $a_{\mid \sigma}<0$. Since $\tau$ is maximal, $\tau_{1}<\tau$ and so $a_{\mid \tau_{1}} \geq 0$. We deduce from $a_{\mid \tau_{1}} \geq 0$ and $a_{\mid \sigma}<0$ that there exists $x \in H\left(\tau_{1}, \sigma\right)$ such that $a(x)=0$. Likewise, there exists $y \in H\left(\tau_{1}^{\prime}, \sigma\right)$ such that $a(y)=0$. Let $\varphi:[0,1] \rightarrow$ BT be a geodesic between $x$ and $y$. Since $x, y \in H(\tau, \sigma)$, $\varphi([0,1]) \subseteq H(\tau, \sigma)$. Each $\varphi(t)$ is an interior point of a polysimplex $\omega(t)$. The map $t \mapsto \omega(t)$ is piecewise constant, so take $0=t_{0}<t_{2}<\cdots<t_{2 k-2}<t_{2 k}=1$ the instants where there is a jump of the map $\omega(t)$. Choose also $t_{1}, \cdots, t_{2 k-1}$ such that $t_{0}<t_{1}<t_{2}<\cdots<t_{2 k-1}<t_{2 k}$. Let $\omega_{i}=\omega\left(t_{i}\right)$. Then $\omega_{2 i}$ and $\omega_{2 i+2}$ must be faces of $\omega_{2 i+1}$ and so for all $i$ we get either $\omega_{i} \leq \omega_{i+1}$ or $\omega_{i+1} \leq \omega_{i}$. Now $a(x)=a(y)=0$ so for all $t, a(\varphi(t))=0$. Hence $\omega_{i}$ contains an interior point $\varphi\left(t_{i}\right)$ such that $a\left(\varphi\left(t_{i}\right)\right)=0$. Lemma 3.13 ensures that $\rho\left(\omega_{i}, \sigma\right)<\rho(\tau, \sigma)$ and $\rho\left(\tau, \omega_{i}\right)<\rho(\tau, \sigma)$. To summarize, we have just built a sequence of polysimplices $\omega_{0}, \cdots, \omega_{2 k}$ such that for all $i, \omega_{i} \in H(\tau, \sigma), \rho\left(\omega_{i}, \sigma\right)<\rho(\tau, \sigma), \rho\left(\tau, \omega_{i}\right)<\rho(\tau, \sigma)$, $\omega_{i} \leq \omega_{i+1}$ or $\omega_{i+1} \leq \omega_{i}, \omega_{0} \in H\left(\tau_{1}, \sigma\right)$ and $\omega_{2 k} \in H\left(\tau_{1}^{\prime}, \sigma\right)$.

Let $i \in\{0,1, \cdots, 2 k\}$. Since $\omega_{i} \in H(\tau, \sigma)$ take, thanks to Lemma 2.4 an admissible path $\tau_{0}^{i}=\tau, \cdots, \tau_{l_{i}}^{i}=\omega_{i}$ from $\tau$ to $\omega_{i}$ such that for all $j, \omega_{i} \in H\left(\tau_{j}^{i}, \sigma\right)$. With Lemma 3.7 we can assume that the $\tau_{j}^{i}$ satisfy the conditions of the latter. We complete the path $\tau_{0}^{i}, \cdots, \tau_{l_{i}}^{i}$ in an admissible path $\tau_{0}^{i}, \cdots, \tau_{n_{i}}^{i}$ between $\tau$ and $\sigma$ thanks to Lemma 2.3 (2). We also assume that $\tau_{0}^{i} \neq \tau_{1}^{i}$ by removing the first polysimplices if they are equal. Since $\tau_{0}^{i}=\tau$ is maximal, $\tau_{0}^{i}>\tau_{1}^{i}$. If $\tau_{1}^{i} \neq \omega_{i}$ then condition (3) of Lemma 3.7 tells us that $\rho\left(\tau_{1}^{i}, \sigma\right)<\rho(\tau, \sigma)$. And if $\tau_{1}^{i}=\omega_{i}$ then by construction of $\omega_{i}, \rho\left(\tau_{1}^{i}, \sigma\right)=\rho\left(\omega_{i}, \sigma\right)<\rho(\tau, \sigma)$. We have just constructed an admissible path $\tau_{0}^{i}, \cdots, \tau_{n_{i}}^{i}$ between $\tau$ and $\sigma$ such that $\rho\left(\tau_{1}^{i}, \sigma\right)<\rho(\tau, \sigma), \omega_{i} \in H\left(\tau_{1}^{i}, \sigma\right)$ et $\tau_{1}^{i}<\tau$. Denote by

$$
\varepsilon_{i}:=\varepsilon_{\tau_{n_{i}}^{i}}^{\tau_{n_{i}-1}^{i}} \circ \cdots \circ \varepsilon_{\tau_{1}^{i}}^{\tau_{0}^{i}}
$$

the local map associated to this path.

Let $i \in\{0,1, \cdots, 2 k-1\}$. We have $\omega_{i} \leq \omega_{i+1}$ or $\omega_{i+1} \leq \omega_{i}$. Denote by $\omega$ the smallest of the two polysimplices so that $\omega \leq \omega_{i}$ and $\omega \leq \omega_{i+1}$. Then $\tau_{0}^{i}, \cdots, \tau_{n_{i}}^{i}$ and $\tau_{0}^{i+1}, \cdots, \tau_{n_{i+1}}^{i+1}$ satisfy the conditions of Lemma 3.12. Indeed, $\rho\left(\tau_{1}^{i}, \sigma\right)<\rho(\tau, \sigma)$ and $\rho\left(\tau_{1}^{i+1}, \sigma\right)<\rho(\tau, \sigma)$. Since $\omega_{i} \in H\left(\tau_{1}^{i}, \sigma\right)$ and $\omega \leq \omega_{i}$, we have that $\omega \in H\left(\tau_{1}^{i}, \sigma\right)$, and likewise $\omega \in H\left(\tau_{1}^{i+1}, \sigma\right)$. Then $\rho\left(\tau, \omega_{i}\right)<\rho(\tau, \sigma)$ and $\rho\left(\tau, \omega_{i+1}\right)<\rho(\tau, \sigma)$, therefore, since $\omega=\omega_{i}$ or $\omega=\omega_{i+1}, \rho(\tau, \omega)<\rho(\tau, \sigma)$. Finally $\tau_{1}^{i}<\tau$ so $\tau_{1}^{i} \in$ $H(\tau, \omega)$ and likewise $\tau_{1}^{i+1} \in H(\tau, \omega)$. Hence Lemma 3.12 tells us that $\varepsilon_{i}=\varepsilon_{i+1}$. This being true for every $i \in\{0, \cdots, 2 k-1\}$ we get $\varepsilon_{0}=\varepsilon_{2 k}$.

The admissible path $\tau_{0}, \cdots, \tau_{n}$ and $\tau_{0}^{0}, \cdots, \tau_{n_{0}}^{0}$ also satisfy the conditions of 3.12 with $\omega=\omega_{0}$ so $\varepsilon_{\tau_{n}}^{\tau_{n-1}} \circ \cdots \circ \varepsilon_{\tau_{1}}^{\tau_{0}}=\varepsilon_{0}$. It is the same with $\tau_{0}^{\prime}, \cdots, \tau_{m}^{\prime}$ and $\tau_{0}^{2 k}, \cdots, \tau_{n_{2 k}}^{2 k}$ by taking $\omega=\omega_{2 k}$ so $\varepsilon_{\tau_{m}^{\prime}}^{\tau_{m-1}^{\prime}} \circ \cdots \circ \varepsilon_{\tau_{1}^{\prime}}^{\tau_{0}^{\prime}}=\varepsilon_{2 k}$. We finally find that

$$
\varepsilon_{\tau_{n}}^{\tau_{n-1}} \circ \cdots \circ \varepsilon_{\tau_{1}}^{\tau_{0}}=\varepsilon_{\tau_{m}^{\prime}}^{\tau_{m-1}^{\prime}} \circ \cdots \circ \varepsilon_{\tau_{1}^{\prime}}^{\tau_{0}^{\prime}}
$$

which completes the proof. 
Now that we have shown that these local maps are well defined (using admissible paths) it is easy to check that they satisfy some good properties. In particular, we have the following proposition.

3.15. Proposition. Let $\tau, \sigma, \omega \in \mathrm{BT}$ such that $\omega \in H(\tau, \sigma)$. Then

$$
\varepsilon_{\sigma}^{\tau}=\varepsilon_{\sigma}^{\omega} \circ \varepsilon_{\omega}^{\tau} .
$$

Proof. Proposition 3.14 tells us that $\varepsilon_{\sigma}^{\tau}$ is independent of the chosen admissible path. All we have to do is then take, thanks to Lemma 2.4 an admissible path between $\tau$ and $\sigma$ going though $\omega$ such that the two paths from $\tau$ to $\omega$ and from $\omega$ to $\sigma$ are admissible.

3.16. Remark. Proposition 3.15 proves Lemmas (2.2.8), (2.2.9) and (2.2.10) of Wan17.

\section{Equivalence of CATEgories}

In this section, we finish the proof of the two theorems stated in the introduction. In the previous section, we have shown how to define the local maps $\varepsilon_{\sigma}^{\tau}$ in the case of any reductive group, and that these maps satisfy the same properties as in Wan17. Hence, we can follow Section 2.3 and 2.4 in Wan17] to get the result. For the reader's convenience, we will recall here the proof. We will refer to Wan17. and MS10] for full details of the proofs.

Let $\Gamma=\left(V_{\sigma}\right)_{\sigma \in \mathrm{BT}}$ be a $e$-coefficient system. Let $\Sigma$ be a finite convex subcomplex of BT. Fix a vertex $x \in \Sigma$ and denote by $\underline{V_{x}}:=\left(\sigma \mapsto V_{x}, \varphi_{x}^{\sigma}=\operatorname{Id}_{V_{x}}\right)$ the constant coefficient system with value in $V_{x}$. The local maps $\left\{\varepsilon_{x}^{\sigma}\right\}_{\sigma \in \Sigma}$ induce a morphism of coefficient system and so of chain complexes

$$
\oplus_{\sigma \in \Sigma} \varepsilon_{x}^{\sigma}: C_{*}(\Sigma, \Gamma) \rightarrow C_{*}\left(\Sigma, \underline{V_{x}}\right)
$$

and a morphism on homologies

$$
p_{x}^{\Sigma}:=H_{0}\left(\oplus \varepsilon_{x}^{\sigma}\right): H_{0}(\Sigma, \Gamma) \rightarrow H_{0}\left(\Sigma, \underline{V_{x}}\right) .
$$

Let us remark, that since $\Sigma$ is finite convex, it is contractible, thus $C_{*}\left(\Sigma, \underline{V_{x}}\right)$ is a resolution of $V_{x}$. In particular, $H_{0}\left(\Sigma, V_{x}\right)=V_{x}$ and $p_{x}^{\Sigma}: H_{0}(\Sigma, \Gamma) \rightarrow V_{x}$.

Let $\Sigma^{\prime} \subseteq \Sigma$ be a finite convex subcomplex of $\Sigma$. We have a morphism of complexes

$$
\oplus_{\sigma \in \Sigma^{\prime}} \operatorname{Id}_{V_{\sigma}}: C_{*}\left(\Sigma^{\prime}, \Gamma\right) \rightarrow C_{*}(\Sigma, \Gamma)
$$

and a morphism on homologies

$$
i_{\Sigma^{\prime}}^{\Sigma}:=H_{0}\left(\oplus \operatorname{Id}_{V_{\sigma}}\right): H_{0}\left(\Sigma^{\prime}, \Gamma\right) \rightarrow H_{0}(\Sigma, \Gamma)
$$

From the definition of $p_{x}^{\Sigma}$ it follows the next lemma.

4.1. Lemma (Wan17, Lem. 2.3.1]). The composition of the homology morphisms

$$
H_{0}(\{y\}, \Gamma)=V_{y} \stackrel{i_{y}^{\Sigma}}{\longrightarrow} H_{0}(\Sigma, \Gamma) \stackrel{p_{x}^{\Sigma}}{\longrightarrow} H_{0}\left(\Sigma, \underline{V_{x}}\right)=V_{x}
$$

is $\varepsilon_{x}^{y}$. In particular $p_{x}^{\Sigma} \circ i_{x}^{\Sigma}=\operatorname{Id}_{V_{x}}$.

Thus we can define an idempotent $e_{x}^{\Sigma}$ by

$$
e_{x}^{\Sigma}: H_{0}(\Sigma, \Gamma) \stackrel{p_{x}^{\Sigma}}{\longrightarrow} V_{x} \stackrel{i_{x}^{\Sigma}}{\longrightarrow} H_{0}(\Sigma, \Gamma) .
$$


The properties of $\varepsilon_{\sigma}^{\tau}$ proved in Proposition 3.15 allows us to show that the idempotents $e_{x}^{\Sigma}$ satisfies the consistency properties of [MS10. Denote by $\Sigma_{0}$ the subset of $\Sigma$ of vertices.

4.2. Proposition. We have the following properties:

(1) If $x, y, z \in \Sigma_{0}, z, x$ are adjacent and $z \in H(x, y)$, then $e_{x}^{\Sigma} \circ e_{z}^{\Sigma} \circ e_{y}^{\Sigma}=e_{x}^{\Sigma} \circ e_{y}^{\Sigma}$.

(2) If $x, y \in \Sigma_{0}$ are adjacent, then $e_{x}^{\Sigma} \circ e_{y}^{\Sigma}=e_{y}^{\Sigma} \circ e_{x}^{\Sigma}$.

Proof. We follow the proof of Wan17, Lem. 2.3.2]. We just need to adapt a little bit the proof of (2), because, for a general building, $[x, y]$ does not necessarily have dimension 1.

Since $H_{0}(\Sigma, \Gamma)=\sum_{w \in \Sigma_{0}} i_{w}^{\Sigma}\left(V_{w}\right)$, it is enough to prove that for all $w \in \Sigma_{0}$, $e_{x}^{\Sigma} \circ e_{y}^{\Sigma}=e_{y}^{\Sigma} \circ e_{x}^{\Sigma}\left(\operatorname{resp} . e_{x}^{\Sigma} \circ e_{z}^{\Sigma} \circ e_{y}^{\Sigma}=e_{x}^{\Sigma} \circ e_{y}^{\Sigma}\right)$ on $i_{w}^{\Sigma}\left(V_{w}\right)$.

Let us start by (1). By Lemma 4.1, we have

$$
e_{x}^{\Sigma} \circ e_{z}^{\Sigma} \circ e_{y}^{\Sigma} \circ i_{w}^{\Sigma}=i_{x}^{\Sigma} \circ p_{x}^{\Sigma} \circ i_{z}^{\Sigma} \circ p_{z}^{\Sigma} \circ i_{y}^{\Sigma} \circ p_{y}^{\Sigma} \circ i_{w}^{\Sigma}=i_{x}^{\Sigma} \circ \varepsilon_{x}^{z} \circ \varepsilon_{z}^{y} \circ \varepsilon_{y}^{w}
$$

and

$$
e_{x}^{\Sigma} \circ e_{y}^{\Sigma} \circ i_{w}^{\Sigma}=i_{x}^{\Sigma} \circ p_{x}^{\Sigma} \circ i_{y}^{\Sigma} \circ p_{y}^{\Sigma} \circ i_{w}^{\Sigma}=i_{x}^{\Sigma} \circ \varepsilon_{x}^{y} \circ \varepsilon_{y}^{w}
$$

We obtain the equality with Proposition 3.15 .

Now, we prove (2). Let $a \in V_{w}$. We have

$$
e_{x}^{\Sigma} \circ e_{y}^{\Sigma} \circ i_{w}^{\Sigma}(a)-e_{y}^{\Sigma} \circ e_{x}^{\Sigma} \circ i_{w}^{\Sigma}(a)=i_{x}^{\Sigma} \circ \varepsilon_{x}^{y} \circ \varepsilon_{y}^{w}(a)-i_{y}^{\Sigma} \circ \varepsilon_{y}^{x} \circ \varepsilon_{x}^{w}(a) .
$$

Denote by $i_{x}^{\prime \Sigma}: V_{x} \rightarrow V_{x} \oplus V_{y} \oplus \bigoplus_{s \in \Sigma_{0} \backslash\{x, y\}} V_{s}\left(\right.$ resp. $\quad i_{y}^{\prime \Sigma}: V_{y} \rightarrow V_{x} \oplus V_{y} \oplus$ $\left.\bigoplus_{s \in \Sigma_{0} \backslash\{x, y\}} V_{s}\right)$ be the natural embedding $u \mapsto(u, 0,0, \cdots)($ resp. $v \mapsto(0, v, 0, \cdots))$. We have two commutative diagrams
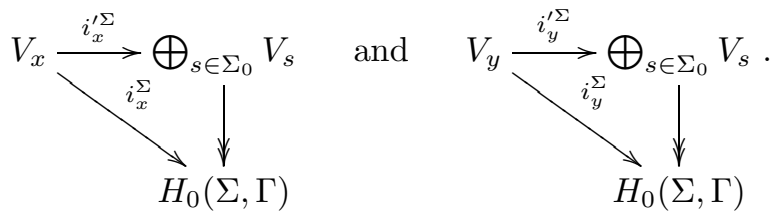

Thus, we need to show that $i_{x}^{\Sigma} \circ \varepsilon_{x}^{y} \circ \varepsilon_{y}^{w}(a)-i_{y}^{\prime \Sigma} \circ \varepsilon_{y}^{x} \circ \varepsilon_{x}^{w}(a) \in \partial\left(\oplus_{\sigma \in \Sigma, \operatorname{dim}(\sigma)=1} V_{\sigma}\right)$. Let $z_{0}:=x, z_{1}, \cdots, z_{r}:=y$ be a sequence of vertices of $[x, y]$ such that for all $0 \leq i \leq r-1, \operatorname{dim}\left(\left[z_{i}, z_{i+1}\right]\right)=1$, and $r$ is minimal. Now we define an element $b \in \oplus_{\sigma \in \Sigma, \operatorname{dim}(\sigma)=1} V_{\sigma}$ by $b_{\left[z_{i}, z_{i+1}\right]}:=\varepsilon_{\left[z_{i}, z_{i+1}\right]}^{[x, y]} \circ \varepsilon_{[x, y]}^{w}(a) \in V_{\left[z_{i}, z_{i+1}\right]}$ and 0 elsewhere. By Lemma 3.15.

$$
\begin{aligned}
\varphi_{x}^{\left[x, z_{1}\right]}\left(b_{\left[x, z_{1}\right]}\right) & =\varepsilon_{x}^{\left[x, z_{1}\right]} \circ \varepsilon_{\left[x, z_{1}\right]}^{[x, y]} \circ \varepsilon_{[x, y]}^{w}(a)=\varepsilon_{x}^{[x, y]} \circ \varepsilon_{[x, y]}^{w}(a) \\
& =\varepsilon_{x}^{[x, y]} \circ \varepsilon_{[x, y]}^{y} \circ \varepsilon_{y}^{w}(a)=\varepsilon_{x}^{y} \circ \varepsilon_{y}^{w}(a) .
\end{aligned}
$$

In the same way,

$$
\varphi_{y}^{\left[y, z_{r-1}\right]}\left(b_{\left[y, z_{r-1}\right]}\right)=\varepsilon_{y}^{\left[y, z_{r-1}\right]} \circ \varepsilon_{\left[y, z_{r-1}\right]}^{[x, y]} \circ \varepsilon_{[x, y]}^{x} \circ \varepsilon_{x}^{w}(a)=\varepsilon_{y}^{x} \circ \varepsilon_{x}^{w}(a) .
$$


Also, for $1 \leq i \leq r-1$,

$$
\begin{aligned}
& \varphi_{z_{i}}^{\left[z_{i}, z_{i+1}\right]}\left(b_{\left[z_{i}, z_{i+1}\right]}\right)=\varepsilon_{z_{i}}^{\left[z_{i}, z_{i+1}\right]} \circ \varepsilon_{\left[z_{i}, z_{i+1}\right]}^{[x, y]} \circ \varepsilon_{[x, y]}^{w}(a)=\varepsilon_{z_{i}}^{[x, y]} \circ \varepsilon_{[x, y]}^{w}(a) \\
& \varphi_{z_{i}}^{\left[z_{i}, z_{i-1}\right]}\left(b_{\left[z_{i}, z_{i-1}\right]}\right)=\varepsilon_{z_{i}}^{\left[z_{i}, z_{i-1}\right]} \circ \varepsilon_{\left[z_{i}, z_{i-1}\right]}^{[x, y]} \circ \varepsilon_{[x, y]}^{w}(a)=\varepsilon_{z_{i}}^{[x, y]} \circ \varepsilon_{[x, y]}^{w}(a)
\end{aligned}
$$

Therefore

$$
\varphi_{z_{i}}^{\left[z_{i}, z_{i+1}\right]}\left(b_{\left[z_{i}, z_{i+1}\right]}\right)-\varphi_{z_{i}}^{\left[z_{i}, z_{i-1}\right]}\left(b_{\left[z_{i}, z_{i-1}\right]}\right)=0 .
$$

Thus, $i_{x}^{\prime \Sigma} \circ \varepsilon_{x}^{y} \circ \varepsilon_{y}^{w}(a)-i_{y}^{\prime \Sigma} \circ \varepsilon_{y}^{x} \circ \varepsilon_{x}^{w}(a)=\partial(b)$ and this finish the proof.

Now, since this system of idempotents satisfies the consistency properties, we can follow the strategy of Meyer and Solleveld. Thanks to property (2), we can define $e_{\sigma}^{\Sigma}:=\prod_{x \leq \sigma, x \in \Sigma_{0}} e_{x}^{\Sigma} \in \operatorname{End}\left(H_{0}(\Sigma, \Gamma)\right)$ and, as in [MS10, Thm. 2.12], $u_{\Sigma^{\prime}}^{\Sigma}:=$ $\sum_{\sigma \in \Sigma^{\prime}}(-1)^{\operatorname{dim}(\sigma)} e_{\sigma}^{\Sigma}$.

4.3. Proposition. We have

$$
\begin{aligned}
u_{\Sigma^{\prime}}^{\Sigma}\left(H_{0}(\Sigma, \Gamma)\right) & =\sum_{x \in \Sigma_{0}^{\prime}} \operatorname{Im}\left(e_{x}^{\Sigma}\right) \\
\operatorname{Ker}\left(u_{\Sigma^{\prime}}^{\Sigma}\right) & =\bigcap_{x \in \Sigma_{0}^{\prime}} \operatorname{Ker}\left(e_{x}^{\Sigma}\right) \\
H_{0}(\Sigma, \Gamma) & =u_{\Sigma^{\prime}}^{\Sigma}\left(H_{0}(\Sigma, \Gamma)\right) \oplus \operatorname{Ker}\left(u_{\Sigma^{\prime}}^{\Sigma}\right)
\end{aligned}
$$

Moreover, if $\Sigma^{\prime}=\Sigma$, then $\operatorname{Ker}\left(u_{\Sigma}^{\Sigma}\right)=\bigcap_{x \in \Sigma_{0}} \operatorname{Ker}\left(e_{x}^{\Sigma}\right)=0$.

Proof. By Proposition 4.2, the system of idempotent $\left(e_{x}^{\Sigma}\right)_{x \in \Sigma_{0}}$ satisfies the consistency properties of [MS10]. Hence the proof of [MS10, Thm. 2.12] applies here. Now if $\Sigma^{\prime}=\Sigma$, then $H_{0}(\Sigma, \Gamma)=\sum_{x \in \Sigma_{0}^{\prime}} i_{x}^{\Sigma}\left(V_{x}\right)=\sum_{x \in \Sigma_{0}^{\prime}} \operatorname{Im}\left(e_{x}^{\Sigma}\right)$ thus $\operatorname{Ker}\left(u_{\Sigma}^{\Sigma}\right)=0$.

4.4. Proposition (Wan17, Prop. 2.4.1 (b)]). The map $i_{\Sigma^{\prime}}^{\Sigma}: H_{0}\left(\Sigma^{\prime}, \Gamma\right) \rightarrow H_{0}(\Sigma, \Gamma)$ is injective.

Proof. We have a commutative diagram

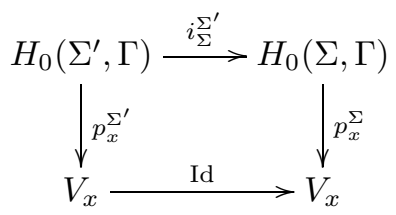

Let $a \in H_{0}\left(\Sigma^{\prime}, \Gamma\right)$ such that $i_{\Sigma}^{\Sigma^{\prime}}(a)=0$. By the previous diagram, for all $x \in \Sigma_{0}^{\prime}$, $p_{x}^{\Sigma^{\prime}}(a)=0$. Thus $e_{x}^{\Sigma^{\prime}}(a)=i_{x}^{\Sigma^{\prime}} \circ p_{x}^{\Sigma^{\prime}}(a)=0$, and $a \in \bigcap_{x \in \Sigma_{0}^{\prime}} \operatorname{Ker}\left(e_{x}^{\Sigma^{\prime}}\right)$. By Proposition 4.3, $a=0$.

4.5. Remark. The proof of [Wan17, Prop. 2.4.1 (b)] also shows that the image of $i_{\Sigma^{\prime}}^{\Sigma}$ is $u_{\Sigma^{\prime}}^{\Sigma}\left(H_{0}(\Sigma, \Gamma)\right)$.

4.6. Lemma. Let $\Sigma$ be a finite convex subcomplex. For all $n>0, H_{n}(\Sigma, \Gamma)=0$.

Proof. As in Wan17, Prop. 2.4.1 (a)] we prove the result by induction.

4.7. Lemma. Let us assume that $\Sigma$ is not a polysimplex and that for all $\Sigma^{\prime} \subsetneq \Sigma$ finite convex and for all $n>0, H_{n}\left(\Sigma^{\prime}, \Gamma\right)=0$. Then for all $n>0, H_{n}(\Sigma, \Gamma)=0$. 
Proof. By [MS10, Section 2.5] we can decompose $\Sigma$ as $\Sigma=\Sigma_{+} \cup \Sigma_{-}$, with $\Sigma_{+}$, $\Sigma_{-}$and $\Sigma_{0}:-\Sigma_{+} \cap \Sigma_{-}$are finite convex proper subcomplexes. The cellular chain complexes for these subcomplexes form an exact sequence

$$
C_{*}\left(\Sigma_{0}, \Gamma\right) \longmapsto C_{*}\left(\Sigma_{+}, \Gamma\right) \oplus C_{*}\left(\Sigma_{-}, \Gamma\right) \rightarrow C_{*}(\Sigma, \Gamma)
$$

which generates a Mayer-Vietoris long exact sequence for their homology groups. Since $\Sigma_{+}, \Sigma_{-}$and $\Sigma_{0}$ satisfies the hypothesis of the Lemma, we get that $H_{n}(\Sigma, \Gamma)=$ 0 for $n \geq 2$. By Proposition 4.4 the map $i_{\Sigma_{0}}^{\Sigma_{+}}: H_{0}\left(\Sigma_{0}, \Gamma\right) \rightarrow H_{0}\left(\Sigma_{+}, \Gamma\right)$ is injective, hence $H_{1}(\Sigma, \Gamma)=0$.

We are left with the case where $\Sigma$ is a single polysimplex. Let $\sigma \subseteq \Sigma$ and $x \in \Sigma_{0}$. We define an idempotent $e_{x}^{\sigma} \in \operatorname{End}\left(V_{\sigma}\right)$ by $e_{x}^{\sigma}:=\varepsilon_{\sigma}^{[x, \sigma]} \circ \varepsilon_{[x, \sigma]}^{\sigma}$. Let $y \in \Sigma_{0}$ (hence $y$ is adjacent to $x$ ) and $z \in H(x, y)$. By Lemma [3.3, $e_{x}^{\sigma} e_{y}^{\sigma}=\varepsilon_{\sigma}^{[x, y, \sigma]} \circ \varepsilon_{[x, y, \sigma]}^{\sigma}=e_{y}^{\sigma} e_{x}^{\sigma}$ and $e_{x}^{\sigma} e_{z}^{\sigma} e_{y}^{\sigma}=\varepsilon_{\sigma}^{[x, y, z, \sigma]} \circ \varepsilon_{[x, y, z, \sigma]}^{\sigma}=\varepsilon_{\sigma}^{[x, y, \sigma]} \circ \varepsilon_{[x, y, \sigma]}^{\sigma}=e_{x}^{\sigma} e_{y}^{\sigma}$. These idempotents satisfies the consistency properties, hence we can follow [MS10, Section 2.5]. For $I \subseteq \Sigma_{0}$, denote by

$$
e_{I}^{\sigma, 0}:=\prod_{x \in I} e_{x}^{\sigma} \prod_{x \notin I}\left(1-e_{x}^{\sigma}\right) \in \operatorname{End}\left(V_{\sigma}\right)
$$

Let $\Sigma_{I}$ be the subcomplex of $\Sigma$ spanned by $I$. As in [MS10, Section 2.5] and Wan17, Prop. 2.4.1 (a)] we have

(1) $e_{I}^{\sigma, 0}\left(V_{\sigma}\right)=0$ if $\sigma_{0} \subsetneq I$

(2) $\operatorname{Id}_{V_{\sigma}}=\sum_{I \subseteq \Sigma_{0}} e_{I}^{\sigma, 0} \in \operatorname{End}\left(V_{\sigma}\right)$

(3) $e_{I}^{\sigma, 0} e_{J}^{\sigma, 0}$, if $I \neq J$

(4) $e_{I}^{\sigma, 0}=0$ if $\Sigma_{I}$ is not a single face of $\Sigma$

Denote by $e_{I}^{0}:=\oplus_{\sigma} e_{I}^{\sigma, 0} \in \operatorname{End}\left(C_{*}(\Sigma, \Gamma)\right)$ the endomorphism on the chain complex, it commutes with the differential. Thus we get a decomposition

$$
C_{*}(\Sigma, \Gamma)=\bigoplus_{I \subseteq \Sigma_{0}} e_{I}^{0}\left(C_{*}(\Sigma, \Gamma)\right) .
$$

If $e_{I}^{0}\left(C_{*}(\Sigma, \Gamma)\right) \neq 0$ then $I \neq \emptyset$ and $\Sigma_{I}$ is a single face of $\Sigma$. The chain complex $e_{I}^{0}\left(C_{*}(\Sigma, \Gamma)\right)$ computes the homology of $\Sigma_{I}$ with constant coefficients in $e_{I}^{0}\left(V_{\Sigma_{I}}\right)$. Since $\Sigma_{I}$ is contractible, $e_{I}^{0}\left(C_{*}(\Sigma, \Gamma)\right)$ is a resolution of $e_{I}^{0}\left(V_{\Sigma_{I}}\right)$ and we get that $H_{n}(\Sigma, \Gamma)=0$ for $n \geq 1$.

We can now prove the first theorem stated in the introduction.

4.8. Theorem. Let e be a consistent system of idempotents satisfying condition (函) and $\Gamma$ a e-coefficient system on $\mathrm{BT}$. Then the chain complex $C_{*}(\mathrm{BT}, \Gamma)$ is exact except in degree 0.

Proof. We follow the proof of $\mathrm{MS10}$, Section 2.5]. Let $\left(\Sigma_{n}\right)_{n \in \mathbb{Z}}$ be an increasing sequence of finite convex subcomplexes of BT such that BT $=\cup_{n} \Sigma_{n}$ and $C_{*}(\mathrm{BT}, \Gamma)=\underline{\lim _{*}} C_{*}\left(\Sigma_{n}, \Gamma\right)$. By Lemma 4.6, for all $n$ the complex

$$
C_{*}\left(\Sigma_{n}, \Gamma\right) \rightarrow H_{0}\left(\Sigma_{n}, \Gamma\right) \rightarrow 0
$$

is exact. The exactness of inductive limits in the category of $R$-modules tells us that $C_{*}(\mathrm{BT}, \Gamma)$ is a resolution of $\lim H_{0}\left(\Sigma_{n}, \Gamma\right)$. That is, for all $n \geq 1, H_{n}(\mathrm{BT}, \Gamma)=0$ and $H_{0}(\mathrm{BT}, \Gamma)=\lim _{\longrightarrow} H_{0}\left(\Sigma_{n}, \overrightarrow{\Gamma)}\right.$. 
Let us now move on to the equivalence of categories. Let $x$ be a vertex. If $\Sigma^{\prime} \subseteq \Sigma$ are two finite convex subcomplexes of BT containing $x$, then we have a commutative diagram:

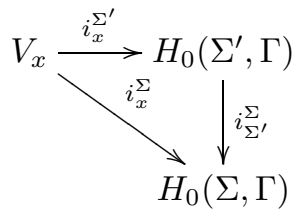

This induces an injection $i_{x}: V_{x} \rightarrow H_{0}(\mathrm{BT}, \Gamma)$.

4.9. Lemma. Let $x$ and $y$ be two adjacent vertices. Then we have a commutative diagram

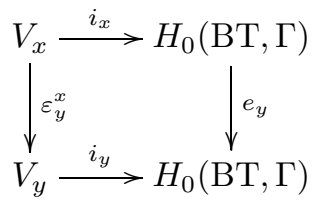

Proof. We follow Wan17, Lem. 2.4.2] and adapt the proof as in Proposition 4.2, Let $i_{x}^{\prime}: V_{x} \rightarrow V_{x} \oplus V_{y} \oplus \bigoplus_{s \in \mathrm{BT}_{0} \backslash\{x, y\}} V_{s}$ and $i_{y}^{\prime}: V_{y} \rightarrow V_{x} \oplus V_{y} \oplus \bigoplus_{s \in \mathrm{BT}_{0} \backslash\{x, y\}} V_{s}$ be the natural embeddings. Let $a \in V_{x}$. We want to show that $e_{y}\left(i_{x}^{\prime}(a)\right)-i_{y}^{\prime}\left(\varepsilon_{y}^{x}(a)\right) \in$ $\partial\left(\bigoplus_{\sigma \in \mathrm{BT}, \operatorname{dim}(\sigma)=1} V_{\sigma}\right)$. Let $z_{0}:=x, z_{1}, \cdots, z_{r}:=y$ be a sequence of vertices of $[x, y]$ such that for all $0 \leq i \leq r-1, \operatorname{dim}\left(\left[z_{i}, z_{i+1}\right]\right)=1$, and $r$ is minimal. Now we define an element $b \in \oplus_{\sigma \in \mathrm{BT}, \operatorname{dim}(\sigma)=1} V_{\sigma}$ by $b_{\left[z_{i}, z_{i+1}\right]}:=\varepsilon_{\left[z_{i}, z_{i+1}\right]}^{[x, y]} \circ \varepsilon_{[x, y]}^{x}(a) \in V_{\left[z_{i}, z_{i+1}\right]}$ and 0 elsewhere. We are using repeatedly Lemma 3.15

$$
\begin{aligned}
\varphi_{x}^{\left[x, z_{1}\right]}\left(b_{\left[x, z_{1}\right]}\right) & =\varepsilon_{x}^{\left[x, z_{1}\right]} \circ \varepsilon_{\left[x, z_{1}\right]}^{[x, y]} \circ \varepsilon_{[x, y]}^{x}(a)=\varepsilon_{x}^{[x, y]} \circ \varepsilon_{[x, y]}^{x}(a) \\
& =e_{[x, y]}(a)=e_{y} e_{x}(a)=e_{y}(a) .
\end{aligned}
$$

In the same way,

$$
\varphi_{y}^{\left[y, z_{r-1}\right]}\left(b_{\left[y, z_{r-1}\right]}\right)=\varepsilon_{y}^{\left[y, z_{r-1}\right]} \circ \varepsilon_{\left[y, z_{r-1}\right]}^{[x, y]} \circ \varepsilon_{[x, y]}^{x}(a)=\varepsilon_{y}^{x}(a) .
$$

Also, for $1 \leq i \leq r-1$,

$$
\begin{aligned}
& \varphi_{z_{i}}^{\left[z_{i}, z_{i+1}\right]}\left(b_{\left[z_{i}, z_{i+1}\right]}\right)=\varepsilon_{z_{i}}^{\left[z_{i}, z_{i+1}\right]} \circ \varepsilon_{\left[z_{i}, z_{i+1}\right]}^{[x, y]} \circ \varepsilon_{[x, y]}^{x}(a)=\varepsilon_{z_{i}}^{[x, y]} \circ \varepsilon_{[x, y]}^{x}(a) \\
& \varphi_{z_{i}}^{\left[z_{i}, z_{i-1}\right]}\left(b_{\left[z_{i}, z_{i-1}\right]}\right)=\varepsilon_{z_{i}}^{\left[z_{i}, z_{i-1}\right]} \circ \varepsilon_{\left[z_{i}, z_{i-1}\right]}^{[x, y]} \circ \varepsilon_{[x, y]}^{x}(a)=\varepsilon_{z_{i}}^{[x, y]} \circ \varepsilon_{[x, y]}^{x}(a)
\end{aligned}
$$

therefore

$$
\varphi_{z_{i}}^{\left[z_{i}, z_{i+1}\right]}\left(b_{\left[z_{i}, z_{i+1}\right]}\right)-\varphi_{z_{i}}^{\left[z_{i}, z_{i-1}\right]}\left(b_{\left[z_{i}, z_{i-1}\right]}\right)=0 .
$$

Thus $e_{y}\left(i_{x}^{\prime}(a)\right)-i_{y}^{\prime}\left(\varepsilon_{y}^{x}(a)\right)=\partial(b)$.

4.10. Theorem. Let e be a consistent system of idempotents satisfying condition (荬) and $\Gamma$ a e-coefficient system on $\mathrm{BT}$. Then $\Gamma$ is isomorphic to the coefficient system $\left(\sigma \mapsto e_{\sigma}\left(H_{0}(\mathrm{BT}, \Gamma)\right)\right)$.

Proof. The proof is the same as in Wan17, Section 2.4]. We recall it here for the reader's convenience. By Lemma 4.9, the morphism $i_{x}$ has its image in $e_{x}\left(H_{0}(\mathrm{BT}, \Gamma)\right)$. Let $\sigma$ be a polysimplex containing $x$ and $i_{\sigma}:=i_{x \mid e_{\sigma}\left(V_{x}\right)}$. The morphism $i_{\sigma}$ sends $\varphi_{x}^{\sigma}\left(V_{\sigma}\right)$ into $e_{\sigma}\left(H_{0}(\mathrm{BT}, \Gamma)\right)$, and is independent of the choice of the vertex $x$ contained in $\sigma$. Thus the morphisms $\left\{i_{\sigma}\right\}_{\sigma \in \mathrm{BT}}$ induce a morphism of coefficient systems

$$
\Gamma \rightarrow\left(\sigma \mapsto e_{\sigma}\left(H_{0}(\mathrm{BT}, \Gamma)\right)\right)_{\sigma \in \mathrm{BT}} .
$$


Since $H_{0}(\mathrm{BT}, \Gamma)=\sum_{y \in \mathrm{BT}_{0}} i_{y}\left(V_{y}\right)$ it is enough to prove that for all $y \in \mathrm{BT}_{0}$, $e_{\sigma}\left(i_{y}\left(V_{y}\right)\right) \subseteq i_{x}\left(V_{\sigma}\right)$. Let $y \in \mathrm{BT}_{0}$. Now take a sequence of vertices $z_{0}=$ $y, z_{1}, \cdots, z_{m}=x$ such that $z_{i}$ and $z_{i+1}$ are adjacent, and $z_{i+1} \in H\left(z_{i}, x\right)$. Then

$$
e_{\sigma}\left(i_{y}\left(V_{y}\right)\right)=e_{\sigma} e_{y}\left(i_{y}\left(V_{y}\right)\right)=e_{\sigma} e_{z_{1}} e_{y}\left(i_{y}\left(V_{y}\right)\right)=\cdots=e_{\sigma} e_{z_{m}} \cdots e_{z_{1}} e_{y}\left(i_{y}\left(V_{y}\right)\right) .
$$

By Lemma 4.9

$$
\begin{aligned}
e_{\sigma} e_{z_{m}} \cdots e_{z_{1}} e_{y}\left(i_{y}\left(V_{y}\right)\right) & =e_{\sigma} e_{z_{m}} \cdots e_{z_{2}} i_{z_{1}}\left(\varepsilon_{z_{1}}^{y}\left(V_{y}\right)\right) \subseteq e_{\sigma} e_{z_{m}} \cdots e_{z_{2}} i_{z_{1}}\left(V_{z_{1}}\right) \\
& =e_{\sigma} e_{z_{m}} \cdots e_{z_{2}} i_{z_{2}}\left(\varepsilon_{z_{2}}^{z_{1}}\left(V_{z_{1}}\right) \subseteq \subseteq e_{\sigma} e_{z_{m}} \cdots i_{z_{2}}\left(V_{z_{2}}\right)\right. \\
& \subseteq \cdots \subseteq e_{\sigma} i_{x}\left(V_{x}\right)=i_{x}\left(V_{\sigma}\right)
\end{aligned}
$$

Let $e$ be a system of idempotents and $V$ a smooth $R G$-module. We define a coefficient system $\Gamma(V)$ by $V_{\sigma}:=e_{\sigma} V$ and for $\tau \leq \sigma, \varphi_{\sigma}^{\tau}$ is the inclusion $V_{\sigma} \hookrightarrow V_{\tau}$.

4.11. Theorem. Let e be a consistent system of idempotents satisfying the condition (函. Then the functor

$$
\begin{array}{ccc}
\operatorname{Rep}_{R}^{e}(G) & \rightarrow & \operatorname{Coef}_{e}(G, R) \\
V & \mapsto & \Gamma(V)
\end{array}
$$

admits a quasi-inverse $\Gamma \mapsto H_{0}(\mathrm{BT}, \Gamma)$, hence induces an equivalence of categories.

Proof. This is done in [Wan17, Cor (2.1.11)]. By Theorem 4.10, the functor $V \mapsto$ $\Gamma(V)$ is essentially surjective. Hence, we need to prove that it is fully faithful.

For $V, W \in \operatorname{Rep}_{R}^{e}(G), \Gamma$ induces a morphism

$$
\begin{array}{ccc}
\Gamma: \operatorname{Hom}_{R G}(V, W) & \rightarrow \operatorname{Hom}_{\mathrm{Coef}_{e}}(\Gamma(V), \Gamma(W)) \\
f & \mapsto\left(f_{\sigma}=f_{\mid e_{\sigma}(V)}: V_{\sigma} \rightarrow W_{\sigma}\right)
\end{array} .
$$

Let $f \in \operatorname{Hom}_{R G}(V, W)$ such that $\Gamma(f)=0$. Then for all vertex $x, f_{\mid e_{x}(V)}=0$. Since $V \in \operatorname{Rep}_{R}^{e}(G), V=\sum_{x \in \mathrm{BT}_{0}} e_{x}(V)$ and $f=0$. Hence the injectivity of $\Gamma$. For the surjectivity, let $\left(g_{\sigma}\right)_{\sigma \in \mathrm{BT}} \in \operatorname{Hom}_{\mathrm{Coef}_{e}}(\Gamma(V), \Gamma(W))$. We get a morphism of complexes

$$
g: C_{*}(\mathrm{BT}, \Gamma(V)) \rightarrow C_{*}(\mathrm{BT}, \Gamma(W)) .
$$

By MS10, Thm. 2.4] $C_{*}(\mathrm{BT}, \Gamma(V)) \rightarrow V \rightarrow 0$ (resp. $C_{*}(\mathrm{BT}, \Gamma(W)) \rightarrow W \rightarrow 0$ ) is a resolution of $V$ (resp. W). Hence $H_{0}(g)$ induces a morphism of $R G$-modules

$$
H_{0}(g): V \rightarrow W
$$

By definition, for every $x \in \mathrm{BT}_{0}$, we have $H_{0}(g)_{\mid e_{x}(V)}=g_{x}$. Hence for every $\sigma \in \mathrm{BT}$ containing $x, H_{0}(g)_{\mid e_{\sigma}(V)}=\left(H_{0}(g)_{\mid e_{x}(V)}\right)_{\mid e_{\sigma}(V)}=g_{x \mid e_{\sigma}(V)}=g_{\sigma}$. And we have the surjectivity.

\section{REFERENCES}

[BT72] F. Bruhat and J. Tits, Groupes réductifs sur un corps local (French), Inst. Hautes Études Sci. Publ. Math. 41 (1972), 5-251. MR.327923

[BT84] F. Bruhat and J. Tits, Groupes réductifs sur un corps local. II. Schémas en groupes. Existence d'une donnée radicielle valuée, Inst. Hautes Études Sci. Publ. Math. (1984), no. 60, 197-376. MR756316 (86c:20042)

[Dat18] Jean-François Dat, Equivalences of tame blocks for p-adic linear groups, Math. Ann. 371 (2018), no. 1-2, 565-613, DOI 10.1007/s00208-018-1648-1. MR3788858

[Lan18a] Thomas Lanard, Sur les $\ell$-blocs de niveau zéro des groupes p-adiques (French, with English summary), Compos. Math. 154 (2018), no. 7, 1473-1507, DOI 10.1112/s0010437x18007133. MR3826462 
[Lan18b] Thomas Lanard, Sur les $\ell$-blocs de niveau zéro des groupes p-adiques II, arXiv e-prints (2018), arXiv:1806.09543.

[MS10] Ralf Meyer and Maarten Solleveld, Resolutions for representations of reductive $p$ adic groups via their buildings, J. Reine Angew. Math. 647 (2010), 115-150, DOI 10.1515/CRELLE.2010.075. MR2729360

[SS97] Peter Schneider and Ulrich Stuhler, Representation theory and sheaves on the BruhatTits building, Inst. Hautes Études Sci. Publ. Math. 85 (1997), 97-191. MR1471867

[Vig96] Marie-France Vignéras, Représentations l-modulaires d'un groupe réductif p-adique avec $l \neq p$ (French, with English summary), Progress in Mathematics, vol. 137, Birkhäuser Boston, Inc., Boston, MA, 1996. MR1395151

[Vig97] Marie-France Vignéras, Cohomology of sheaves on the building and R-representations, Invent. Math. 127 (1997), no. 2, 349-373, DOI 10.1007/s002220050124. MR1427623

[Wan17] Haoran Wang, L'espace symétrique de Drinfeld et correspondance de Langlands locale II (French, with English summary), Math. Ann. 369 (2017), no. 3-4, 1081-1130, DOI 10.1007/s00208-016-1489-8. MR 3713536

Faculty of Mathematics, University of Vienna, Oskar-Morgenstern-Platz 1, 1090 Wien, Austria

Email address: thomas.lanard@univie.ac.at 